\title{
Numerical approach for finite volume three-body interaction
}

\author{
Peng Guo* and Vladimir Gasparian \\ Department of Physics and Engineering, California State University, Bakersfield, California 93311, USA
}

(Received 3 October 2017; published 22 January 2018)

\begin{abstract}
In the present work, we study a numerical approach to one dimensional finite volume three-body interaction, the method is demonstrated by considering a toy model of three spinless particles interacting with pair-wise $\delta$-function potentials. The numerical results are compared with the exact solutions of three spinless bosons interaction when the strength of short-range interactions are set equal for all pairs.
\end{abstract}

DOI: 10.1103/PhysRevD.97.014504

\section{INTRODUCTION}

Three-particle interaction plays an important role in many aspects of hadron/nuclear, atomic, and condensed matter physics. The understanding of three-body dynamics is an essential and key element of many physical processes, such as, the decay of $\eta \rightarrow 3 \pi$ [1-8]. Three-body dynamics in free space has been well studied in the past, many approaches were developed, for instance: relativistic Bethe-Salpeter equations approach [9-11], Faddeev equations method [12-17], and Khuri-Treiman equation approach [18-28]. However, due to the complication of three-body dynamics in general, the finite volume three-body formalism is still in its early developing phase. Recent advances in lattice computation have made the study of hadron scattering possible [29-40]. Unfortunately, because of lacking reliable formalism of three-body interaction in finite volume, the current studies of hadron scattering in lattice QCD are only confined in two-body elastic or inelastic regions. The two-body scattering amplitudes are extracted from the results of lattice QCD calculations by using Lüscher's formula [41] or its extensions to moving frames and inelastic channels [42-54]. A reliable and sensible finite volume three-body formalism is urgently needed in lattice QCD community when the energy levels go above the three-body threshold.

In addition to its application in nuclear/hadron physics, the study of three or more particles either in free space or interacting with periodic potentials also has wide variety of applications and interests in condensed matter physics. For example, the rapid development of semiconductor technology has allowed to manufacture quantum dots (QDs) nanometer sized islands. These new nanostructure materials

*3guo@jlab.org

Published by the American Physical Society under the terms of the Creative Commons Attribution 4.0 International license. Further distribution of this work must maintain attribution to the author(s) and the published article's title, journal citation, and DOI. Funded by SCOAP. have triggered a great interest from both experimental and theoretical points of view [55]. Especially, electrons inside these nano-structures can be controlled experimentally, so it may potentially be applied to the development of materials in quantum computing [56] and spintronics [57]. Moreover, the QDs are considered ideal nano-laboratories to study the physical properties of few-particle systems in reduced dimensional space. In this regard, two-electron systems [58-68] become the simplest arrangement of few-particle systems, which may be served as a starting point to evaluate the correlation effects on the energy band structure of more complicated systems. Some of the simplest and exactly solvable models of one quantum dot with two interacting electrons were studied in the past, e.g., [69], in which the hybridization effects with the states on the leads are also considered. These early studies show that the effect of the electron-electron interaction, in contrast to the case of noninteracting electrons, indeed changes the electron density of states at the Fermi level, and result in nontrivial corrections to the conductivity and negative magnetoresistance in disordered conductors [70]. Another class of systems to which the three and more electron interaction applies and is of special interest in condensed matter physics, is a many body localization phenomena. In these phenomena, the many-body eigenstates of the Hamiltonian are localized, and Anderson localization type of behavior can only be described by the interacting few-particle dynamics (see, e.g., [71,72]).

Many attempts on finite volume three-body interactions were made in recent years [73-88] from different approaches. For instance, quantum field theory based diagrammatic approaches or Faddeev equations based method [73-80,85-88], and the approach by considering the asymptotic form of wave function in configuration space $[83,84]$. Unfortunately, the majority of these developments are still mathematically unfriendly to common users and are not easily tested in practice because of the complication of three-body dynamics. Only a few limited cases of three-body problems can be solved analytically in low dimension, such as McGuire's model in finite volume 
[83]. However, diffraction effects in McGuire's model are all cancelled out [89], thus no new momenta are created over scattering process, though momenta are allowed to be rearranged among three particles. As the consequence, asymptotic form of wave function contains only plane waves, the spherical waves are completely absent due to the cancellation of diffraction effect [84]. The absence of spherical waves simplifies the algebra of finite volume three-body dynamics dramatically, making it possible to finally have the quantization conditions expressed in a simple way analytically [83]. In general cases, the analytic solutions of three-body dynamics are usually not available, even for cases that may seems like simple, such as the pairwise $\delta$-function potentials with unequal strength among pairs [90]. Although, as suggested in [84], given the asymptotic form of wave function, it may be possible to obtain three-body quantization conditions in an analytic form that involves only on-shell scattering amplitudes. Obtaining the analytic asymptotic form of three-body wave function or parametrization of on-shell three-body scattering amplitudes never is an easy task even for "simple cases," such as unequal strength $\delta$-function pair-wise interactions. Therefore, in present work, we aim to obtain a numerical approach to the finite volume three-body problems. Although the explicit and analytic form of quantization conditions are sacrificed and abandoned this way, finite volume three-body problems can be solved numerically and quite reliably without any approximation. Most importantly, the approach is applicable to general cases even when three-body forces are included. To demonstrate the approach, in this work, we consider a simple toy model of three spinless particles interacting with pair-wise $\delta$-function potentials. The exact solutions in finite volume are available at the limit of equal strength $\delta$ function potentials [83], which can be used to test our numerical approach. As will be made clear later on, threebody dynamics is completely determined by Faddeev equations, and wave functions in both free space and finite box can be constructed from the solutions of Faddeev equations. The role of the matching condition of free space and finite volume wave functions is to impose the extra constraints on allowed energy spectra in a finite box and eventually leads to discrete values of energy spectra as the consequence of periodic lattice structure. In this work, instead of aiming to obtain analytic expressions of threebody quantization conditions which may be derived from the matching condition, we propose to search allowed energy spectra numerically by using matching condition directly. Since three-body dynamics is solely determined by Faddeev equations, and is independent of lattice structure of finite box, the Faddeev equations can thus be solved separately by numerical approach, and solutions may be tabulated and stored regardless of the scattering of particles in free space or finite volume. Then, the solutions of Faddeev equations may be used as input into matching condition of finite volume problem to search for allowed discrete energy spectra in a finite box. The strategy of numerical approach is illustrated and tested by a toy model with particles interacting by pair-wise $\delta$-function potentials. The toy model is solved numerically and the results are compared with the exact solutions at the limit of equal strength $\delta$-function potentials among all pairs. At last, we would also like to point out that though our discussion and presentation for finite volume three-body problem was focused on pair-wise short-range interactions, the approach can be applied to three-body problems in general when the three-body force is also included. The strategy of solving finite volume threebody problem in general cases remains the same. A brief discussion of three-body problem including three-body force is presented in Appendix B. For completeness, a short review of Faddeev's approach for pair-wise short-range interaction is also provided in Appendix A.

The paper is organized as follows. In Sec. II, we summarize the formalism of three-particle interaction in finite volume. The numerical approach and results are presented in Sec. III. The summary and discussion are given in Sec. IV.

\section{THREE-BODY INTERACTION FOR SHORT RANGE INTERACTION}

\section{A. Three-body interaction in free space}

In this work, for the purpose of demonstration of numerical approach, we consider a nonrelativistic toy model of three-body interaction in one spatial dimension, and assume that all particles are spinless and have equal mass. These assumptions are not essential for physics that we are interested in but only to simplify the algebra and presentation. The interactions among particles are assumed pairwise and are described by $\delta$-function potentials with strength, $V_{\alpha \beta}$, between $\alpha$-th and $\beta$-th particles. The threeparticle wave function satisfies Schrödinger equation,

$$
\begin{aligned}
& {\left[-\frac{1}{2 m} \sum_{i=1}^{3} \frac{d^{2}}{d x_{i}^{2}}+V_{12} \delta\left(r_{12}\right)+V_{23} \delta\left(r_{23}\right)+V_{31} \delta\left(r_{31}\right)-E\right]} \\
& \quad \times \Psi\left(x_{1}, x_{2}, x_{3} ; p_{1}, p_{2}, p_{3}\right)=0 .
\end{aligned}
$$

The three-body problem with pairwise interactions in free space can be handled by the well-known Faddeev's approach $[12,13]$. In this way, the scattering with either free-three-particle or two-body bound state plus third particle in both initial and final states is treated in the same framework. The details of complete derivations of Faddeev's approach for both the scattering of free-threeparticle and scattering on a bound state are listed in Appendix A for the completeness of presentation. A brief discussion of three-body problems with three-body forces is also provided in Appendix B. Hence, only some key results and equations are presented in this section. As proposed in $[12,13]$, the three-body wave function has the form of $\Psi=\Psi_{(0)}+\sum_{\gamma=1}^{3} \Psi_{(\gamma)}$ if initial state is 
free-three-particle, and $\Psi=\sum_{\gamma=1}^{3} \Psi_{(\gamma)}$ for scattering of third particle on a two-body bound state. The relative wave functions, $e^{-i P R} \Psi_{(\gamma)}=\psi_{(\gamma)}\left(r_{\alpha \beta}, r_{\gamma} ; q_{i j}, q_{k}\right)$, are determined by

$$
\begin{aligned}
& \psi_{(\gamma)}\left(r_{\alpha \beta}, r_{\gamma} ; q_{i j}, q_{k}\right)= \psi_{(\gamma)}^{(i n)}\left(r_{\alpha \beta}, r_{\gamma} ; q_{i j}, q_{k}\right) \\
&+\int_{-\infty}^{\infty} \frac{d k}{2 \pi} e^{i \sqrt{\sigma^{2}-\frac{3}{4} k^{2}}\left|r_{\alpha \beta}\right|} e^{i k r_{\gamma}} \\
& \times i t_{\alpha \beta}\left(\sqrt{\sigma^{2}-\frac{3}{4} k^{2}}\right) g_{(\gamma)}\left(k ; q_{i j}, q_{k}\right), \\
& \alpha \neq \beta \neq \gamma,
\end{aligned}
$$

where $t_{\alpha \beta}(k)=-\frac{m V_{\alpha \beta}}{2 k+i m V_{\alpha \beta}}$ is the two-body scattering amplitude in pair $(\alpha \beta)$, and $\sigma^{2}=m E-\frac{P^{2}}{6}=q_{i j}^{2}+\frac{3}{4} q_{k}^{2} \cdot \psi_{(\gamma)}^{(i n)}$ is associated to the incoming wave. If initial state is freethree-particle state, it is given by

$$
\begin{aligned}
\psi_{(\gamma)}^{(i n)}\left(r_{\alpha \beta}, r_{\gamma} ; q_{i j}, q_{k}\right) & \\
= & \int_{-\infty}^{\infty} \frac{d k}{2 \pi} e^{i \sqrt{\sigma^{2}-\frac{3}{4} k^{2}}\left|r_{\alpha \beta}\right|} e^{i k r_{\gamma}} i t_{\alpha \beta}\left(\sqrt{\sigma^{2}-\frac{3}{4} k^{2}}\right) \\
& \times \int_{-\infty}^{\infty} d r_{\gamma}^{\prime} e^{-i k r_{\gamma}^{\prime}} \psi_{(0)}\left(0, r_{\gamma}^{\prime} ; q_{i j}, q_{k}\right) .
\end{aligned}
$$

If the initial state is incident of ith particle on a bound state of pair $(j k)$, it is thus given by

$$
\psi_{(\gamma)}^{(i n)}\left(r_{\alpha \beta}, r_{\gamma} ; q_{i j}, q_{k}\right)=\delta_{\gamma, i} \phi_{(\gamma)}^{B}\left(r_{\alpha \beta}\right) e^{i q_{i}^{B} r_{\gamma}},
$$

where $\phi_{(\gamma)}^{B}\left(r_{\alpha \beta}\right)=\sqrt{-\frac{m V_{j k}}{2}} e^{\frac{m V_{j k}}{2}}\left|r_{\alpha \beta}\right|$ refers to the two-body bound state wave function in pair $(j k)$, and $q_{i}^{B}=$ $\sqrt{\sigma^{2}+\frac{3}{4}\left(\frac{m V_{j k}}{2}\right)^{2}}$. In either case, the $g_{(\gamma)}$ amplitudes satisfy Faddeev type integral equations. In a matrix form, the integral equations for $g_{(\gamma)}$ amplitudes are given by

$$
\begin{aligned}
G(k)= & G^{(0)}(k)+i \int_{-\infty}^{\infty} \frac{d q}{2 \pi} \frac{2 \sqrt{\sigma^{2}-\frac{3}{4} q^{2}}}{\sigma^{2}-\frac{3}{4} q^{2}-\left(k+\frac{q}{2}\right)^{2}+i \epsilon} \\
& \times \mathcal{K}\left(\sqrt{\sigma^{2}-\frac{3}{4} q^{2}}\right) G(q),
\end{aligned}
$$

where $G$ and $G^{(0)}$ stand for column vectors $\left(g_{(3)}, g_{(1)}, g_{(2)}\right)^{T}$ and $\left(g_{(3)}^{(0)}, g_{(1)}^{(0)}, g_{(2)}^{(0)}\right)^{T}$ respectively. The matrix $\mathcal{K}$ is given by Eq. (A24),

$$
\mathcal{K}(q)=\left[\begin{array}{ccc}
0 & i t_{23}(q) & i t_{31}(q) \\
i t_{12}(q) & 0 & i t_{31}(q) \\
i t_{12}(q) & i t_{23}(q) & 0
\end{array}\right],
$$

and $g_{(\gamma)}^{(0)}$, s are defined by incoming waves, for an incoming wave of free-three-particle, we have

$$
\begin{aligned}
g_{(\gamma)}^{(0)}\left(k ; q_{i j}, q_{k}\right)= & i \int_{-\infty}^{\infty} \frac{d q}{2 \pi} \frac{2 \sqrt{\sigma^{2}-\frac{3}{4} q^{2}}}{\sigma^{2}-\frac{3}{4} q^{2}-\left(k+\frac{q}{2}\right)^{2}+i \epsilon}\left[i t_{\beta \gamma}\left(\sqrt{\sigma^{2}-\frac{3}{4} q^{2}}\right) \int_{-\infty}^{\infty} d r_{\alpha}^{\prime} e^{-i q r_{\alpha}^{\prime}} \psi_{(0)}\left(0, r_{\alpha}^{\prime} ; q_{i j}, q_{k}\right)\right. \\
& \left.+i t_{\gamma \alpha}\left(\sqrt{\sigma^{2}-\frac{3}{4} q^{2}}\right) \int_{-\infty}^{\infty} d r_{\beta}^{\prime} e^{-i q r_{\beta}^{\prime}} \psi_{(0)}\left(0, r_{\beta}^{\prime} ; q_{i j}, q_{k}\right)\right] .
\end{aligned}
$$

For scattering of ith particle by a bound state in pair $(j k)$,

$$
\begin{aligned}
g_{(\gamma)}^{(0)}\left(k ; q_{i j}, q_{k}\right)= & \delta_{\alpha, i} \int d r_{\alpha} \phi_{(\alpha)}^{B}\left(r_{\alpha}\right) e^{-i\left(k+\frac{q_{i}^{B}}{2}\right) r_{\alpha}} \\
& +\delta_{\beta, i} \int d r_{\beta} \phi_{(\beta)}^{B}\left(r_{\beta}\right) e^{-i\left(k+\frac{q_{i}^{B}}{2}\right) r_{\beta}} .
\end{aligned}
$$

Faddeev type equations, Eq. (5), have no analytic solutions due to diffraction effects in general, except the special case when the strengths of $\delta$-function potential among all pairs are identical: $V_{12}=V_{23}=V_{31}=V_{0}$, see [83]. Nevertheless, Eq. (5) can be solved numerically rather straightforwardly in general cases, and the numerical solutions of $g_{(\gamma)}$ amplitudes can thus be used as input to construct the free space three-body wave function by Eq. (2). As will be presented next, similarly the finite volume three-body wave function is also constructed by using the solutions of $g_{(\gamma)}$ amplitudes, see Eq. (11).

\section{B. Three-body scattering in finite volume}

When particles are confined in a one dimensional periodic box of the size $L$, as shown in [83], the relative finite volume wave function must satisfy the periodic boundary condition,

$$
\begin{gathered}
\psi^{(L)}\left(r_{\alpha \beta}+n_{\alpha \beta} L, r_{\gamma}+\frac{1}{2} n_{\alpha \beta} L+n_{\beta \gamma} L ; q_{i j}, q_{k}\right) \\
=e^{-i \frac{P}{3} n_{\alpha \beta} L} e^{-i \frac{2 P}{3} n_{\beta \gamma} L} \psi^{(L)}\left(r_{\alpha \beta}, r_{\gamma} ; q_{i j}, q_{k}\right), \\
P=\frac{2 \pi}{L} d, \quad\left(n_{\alpha \beta}, n_{\beta \gamma}, d\right) \in \mathbb{Z} .
\end{gathered}
$$


The finite volume three-body wave function, $\psi^{(L)}$, can be constructed from the three-body free space wave function, $\psi$, by

$$
\begin{aligned}
& \psi^{(L)}\left(r_{\alpha \beta}, r_{\gamma} ; q_{i j}, q_{k}\right) \\
& =\sum_{n_{\alpha \beta}, n_{\beta \gamma} \in \mathbb{Z}} e^{i \frac{P}{3} n_{\alpha \beta} L} e^{i \frac{2 P}{3} n_{\beta \gamma} L} \\
& \quad \times \psi\left(r_{\alpha \beta}+n_{\alpha \beta} L, r_{k}+\frac{1}{2} n_{\alpha \beta} L+n_{\beta \gamma} L ; q_{i j}, q_{k}\right) .
\end{aligned}
$$

The infinite sum may be carried out by using relation

$$
\begin{aligned}
\sum_{n_{\alpha \beta} \in \mathbb{Z}} e^{i\left(\frac{P}{3}+\frac{k}{2}\right) n_{\alpha \beta} L} e^{i \sqrt{\sigma^{2}-\frac{3}{4} k^{2}}\left|r_{\alpha \beta}+n_{\alpha \beta} L\right|} \\
=e^{i \sqrt{\sigma^{2}-\frac{3}{4} k^{2}}\left|r_{\alpha \beta}\right|}+\frac{e^{i \sqrt{\sigma^{2}-\frac{3}{4} k^{2}} r_{\alpha \beta}}}{e^{-i\left(\sqrt{\sigma^{2}-\frac{3}{4} k^{2}}+\frac{P}{3}+\frac{k}{2}\right) L}-1} \\
+\frac{e^{-i \sqrt{\sigma^{2}-\frac{3}{4} k^{2}} r_{\alpha \beta}}}{e^{-i\left(\sqrt{\sigma^{2}-\frac{3}{4} k^{2}}-\frac{P}{3}-\frac{k}{2}\right) L}-1}
\end{aligned}
$$

Using the Poisson summation formula, $\sum_{n_{\beta \gamma} \in \mathbb{Z}} e^{i\left(\frac{2 P}{3}+k\right) n_{\beta \gamma} L}=$ $\frac{2 \pi}{L} \sum_{n \in \mathbb{Z}} \delta\left(\frac{2 P}{3}+k-\frac{2 \pi}{L} n\right)$, and free space three-body wave function in Eq. (2), the finite volume three-body wave function hence yields

$$
\begin{aligned}
& \psi_{(\gamma)}^{(L)}\left(r_{\alpha \beta}, r_{\gamma} ; q_{i j}, q_{k}\right) \\
& =\frac{1}{L} \sum_{n \in \mathbb{Z}}^{k=-\frac{2 P}{3}+\frac{2 \pi n}{L} n}\left[e^{i \sqrt{\sigma^{2}-\frac{3}{4} k^{2}}\left|r_{\alpha \beta}\right|} e^{i k r_{\gamma}}\right. \\
& \left.+\frac{e^{i \sqrt{\sigma^{2}-\frac{3}{4} k^{2}} r_{\alpha \beta}} e^{i k r_{\gamma}}}{e^{-i\left(\sqrt{\sigma^{2}-\frac{3}{4} k^{2}}+\frac{P}{3}+\frac{k}{2}\right) L}-1}+\frac{e^{-i \sqrt{\sigma^{2}-\frac{3}{4} k^{2}} r_{\alpha \beta}} e^{i k r_{\gamma}}}{e^{-i\left(\sqrt{\sigma^{2}-\frac{3}{4} k^{2}}-\frac{P}{3}-\frac{k}{2}\right) L}-1}\right] \\
& \times i t_{\alpha \beta}\left(\sqrt{\sigma^{2}-\frac{3}{4} k^{2}}\right) g_{(\gamma)}\left(k ; q_{i j}, q_{k}\right), \\
& \alpha \neq \beta \neq \gamma \text {. }
\end{aligned}
$$

The quantization conditions that yield the discrete energy spectra for three-body interaction in a finite box may be obtained by matching condition [83],

$$
\begin{aligned}
\sum_{\gamma=1}^{3}\left[\psi_{(\gamma)}^{(L)}\left(r_{\alpha \beta}, r_{\gamma} ; q_{i j}, q_{k}\right)-\psi_{(\gamma)}\left(r_{\alpha \beta}, r_{\gamma} ; q_{i j}, q_{k}\right)\right] \\
\quad= \begin{cases}\psi_{(0)}\left(r_{\alpha \beta}, r_{\gamma} ; q_{i j}, q_{k}\right), & \text { if free-particle initial state, } \\
0, & \text { if incident on a bound state, }\end{cases}
\end{aligned}
$$

where both finite volume wave function, $\psi_{(\gamma)}^{(L)}$ in Eq. (11), and free space wave function, $\psi_{(\gamma)}$ in Eq. (2), are determined by the solutions of Faddeev equations in Eq. (5). In other words, the three-body dynamics is completely described by Faddeev equations, and the role of quantization conditions or matching conditions in Eq. (12) is to impose constraints on allowed energy spectra to meet the requirement of a periodic lattice structure in a finite box. Therefore, given the solution of Faddeev equations of $g$-amplitude, the task is to scan all the possible combinations of $\left(q_{i j}, q_{k}\right)$ to find the solution of energy spectra that fulfill the matching condition in Eq. (12) for an arbitrary pair of $\left(r_{\alpha \beta}, r_{\gamma}\right)$. Bearing this in mind, instead of finding the basis of asymptotic form of wave function in both free space and finite volume [41] and deriving an analytic expression of secular equation from matching conditions [83,84], our strategy is to solve Faddeev equations first, and use the solutions of $g_{(\gamma)}{ }^{-}$ amplitudes as input of matching condition, Eq. (12), to search all possible allowed energy spectra of three-body interaction numerically. Although it seems like the analytic forms of secular equation are lost this way, the numerical approach presented in this work is rather straightforward, and the formalism itself is rather simple and user friendly. The only trade-off is that Faddeev equations have to be solved first numerically, and therefore it is more computationally involved. Fortunately, solving Faddeev equations and searching allowed energy spectra in matching conditions are two independent processes, they can be carried out separately. Therefore in practice, it may be plausible to solve Faddeev equations first for multiple initial momenta and energies, then proceed with the second step of energy spectra searching by using matching condition in Eq. (12). In addition, the procedure and strategy of solving finite volume threebody problems is not limited to only pair-wise interactions, but also could be applied to general cases with three-body forces, see discussion in Appendix B. The idea is demonstrated and compared to exact solutions in next section.

\section{NUMERICAL TEST AND EXACT SOLUTIONS AT THE LIMIT OF $V_{12}=V_{23}=V_{31}=V_{0}$}

\section{A. Scattering of three-boson in general}

Let us consider a totally symmetric free-three-particle incoming wave,

$$
\psi_{(0)}^{\mathrm{sym}}=\sum_{k=1}^{3}\left(e^{i q_{i j} r_{12}}+e^{-i q_{i j} r_{12}}\right) e^{i q_{k} r_{3}} .
$$

At the limit of $V_{12}=V_{23}=V_{31}=V_{0}$, it may describe the scattering of three spinless bosons. Hence, we obtain 


$$
\begin{aligned}
g_{(\gamma)}^{(0)}\left(k ; q_{i j}, q_{k}\right)= & -2 i \frac{2 q_{12}\left[i t_{\beta \gamma}\left(q_{12}\right)+i t_{\gamma \alpha}\left(q_{12}\right)\right]}{\left(k-q_{2}-i \epsilon\right)\left(k-q_{1}+i \epsilon\right)} \\
& +2 i \frac{2 q_{23}\left[i t_{\beta \gamma}\left(-q_{23}\right)+i t_{\gamma \alpha}\left(-q_{23}\right)\right]}{\left(k-q_{2}-i \epsilon\right)\left(k-q_{3}-i \epsilon\right)} \\
& +2 i \frac{2 q_{31}\left[i t_{\beta \gamma}\left(-q_{31}\right)+i t_{\gamma \alpha}\left(-q_{31}\right)\right]}{\left(k-q_{3}-i \epsilon\right)\left(k-q_{1}+i \epsilon\right)} \\
& +4 \pi \delta\left(k-q_{3}\right)\left[i t_{\beta \gamma}\left(-q_{23}\right)+i t_{\gamma \alpha}\left(-q_{23}\right)\right] .
\end{aligned}
$$

Normally, it is more stable numerically to separate the $\delta$ function type singular terms by redefining $g$ 's amplitudes,

$$
\begin{aligned}
g_{(\gamma)}\left(k ; q_{i j}, q_{k}\right)= & \hat{g}_{(\gamma)}\left(k ; q_{i j}, q_{k}\right)+4 \pi \delta\left(k-q_{3}\right) \\
& \times\left[i t_{\beta \gamma}\left(-q_{23}\right)+i t_{\gamma \alpha}\left(-q_{23}\right)\right],
\end{aligned}
$$

where similar to equations of $g_{(\gamma)}$ 's, integral equations for $\hat{g}_{(\gamma)}$ 's are given by,

$$
\begin{aligned}
\hat{G}(k)= & \hat{G}^{(0)}(k)+i \int_{-\infty}^{\infty} \frac{d q}{2 \pi} \frac{2 \sqrt{\sigma^{2}-\frac{3}{4} q^{2}}}{\sigma^{2}-\frac{3}{4} q^{2}-\left(k+\frac{q}{2}\right)^{2}+i \epsilon} \\
& \times \mathcal{K}\left(\sqrt{\sigma^{2}-\frac{3}{4} q^{2}}\right) \hat{G}(q),
\end{aligned}
$$

where $\hat{G}$ and $\hat{G}^{(0)}$ stand for column vectors $\left(\hat{g}_{(3)}, \hat{g}_{(1)}, \hat{g}_{(2)}\right)^{T}$ and $\left(\hat{g}_{(3)}^{(0)}, \hat{g}_{(1)}^{(0)}, \hat{g}_{(2)}^{(0)}\right)^{T}$ respectively, and

$$
\begin{aligned}
\hat{g}_{(\gamma)}^{(0)}( & \left.; q_{i j}, q_{k}\right) \\
= & -2 i \frac{2 q_{12} i t_{\beta \gamma}\left(q_{12}\right)\left[1+i t_{\gamma \alpha}\left(-q_{23}\right)+i t_{\alpha \beta}\left(-q_{23}\right)\right]}{\left(k-q_{2}-i \epsilon\right)\left(k-q_{1}+i \epsilon\right)} \\
& -2 i \frac{2 q_{12} i t_{\gamma \alpha}\left(q_{12}\right)\left[1+i t_{\alpha \beta}\left(-q_{23}\right)+i t_{\beta \gamma}\left(-q_{23}\right)\right]}{\left(k-q_{2}-i \epsilon\right)\left(k-q_{1}+i \epsilon\right)} \\
& +2 i \frac{2 q_{23}\left[i t_{\beta \gamma}\left(-q_{23}\right)+i t_{\gamma \alpha}\left(-q_{23}\right)\right]}{\left(k-q_{2}-i \epsilon\right)\left(k-q_{3}-i \epsilon\right)} \\
& +2 i \frac{2 q_{31}\left[i t_{\beta \gamma}\left(-q_{31}\right)+i t_{\gamma \alpha}\left(-q_{31}\right)\right]}{\left(k-q_{3}-i \epsilon\right)\left(k-q_{1}+i \epsilon\right)} .
\end{aligned}
$$

In terms of $\hat{g}_{(\gamma)}$ amplitudes, the totally symmetric free space and finite volume wave functions are determined respectively by,

$$
\begin{aligned}
& \psi_{(\gamma)}^{\mathrm{sym}}\left(r_{\alpha \beta}, r_{\gamma} ; q_{i j}, q_{k}\right) \\
& =2 i t_{\alpha \beta}\left(q_{12}\right)\left[1+i t_{\beta \gamma}\left(-q_{23}\right)+i t_{\gamma \alpha}\left(-q_{23}\right)\right] e^{i q_{12}\left|r_{\alpha \beta}\right|} e^{i q_{3} r_{\gamma}} \\
& \quad+2 i t_{\alpha \beta}\left(-q_{23}\right) e^{-i q_{23}\left|r_{\alpha \beta}\right|} e^{i q_{1} r_{\gamma}}+2 i t_{\alpha \beta}\left(-q_{31}\right) e^{-i q_{31}\left|r_{\alpha \beta}\right|} e^{i q_{2} r_{\gamma}} \\
& \quad+\int_{-\infty}^{\infty} \frac{d k}{2 \pi} e^{i \sqrt{\sigma^{2}-\frac{3}{4} k^{2}}\left|r_{\alpha \beta}\right|} e^{i k r_{\gamma}} i t_{\alpha \beta}\left(\sqrt{\sigma^{2}-\frac{3}{4} k^{2}}\right) \\
& \quad \times \hat{g}_{(\gamma)}\left(k ; q_{i j}, q_{k}\right), \quad \alpha \neq \beta \neq \gamma .
\end{aligned}
$$

Separating the $\delta$-function type singular terms from $g$ 's by Eq. (15) has no effect on nontrivial solutions of three-body problem in finite volume, thus for nontrivial solutions, finite volume wave function has a similar form to Eq. (11),

$$
\begin{aligned}
\psi_{(\gamma)}^{\operatorname{sym}(L)}\left(r_{\alpha \beta}, r_{\gamma} ; q_{i j}, q_{k}\right) & \frac{1}{L} \sum_{n \in \mathbb{Z}}^{k=\frac{2 P}{3}+\frac{2 \pi}{L} n}\left[e^{i \sqrt{\sigma^{2}-\frac{3}{4} k^{2}}\left|r_{\alpha \beta}\right|} e^{i k r_{\gamma}}\right. \\
& +\frac{e^{i \sqrt{\sigma^{2}-\frac{3}{4} k^{2}} r_{\alpha \beta}} e^{i k r_{\gamma}}}{e^{-i\left(\sqrt{\sigma^{2}-\frac{3}{4} k^{2}}+\frac{P}{3}+\frac{k}{2}\right) L}-1}+\frac{e^{-i \sqrt{\sigma^{2}-\frac{3}{4} k^{2}} r_{\alpha \beta}} e^{i k r_{\gamma}}}{\left.e^{-i\left(\sqrt{\left.\sigma^{2}-\frac{3}{4} k^{2}-\frac{P}{3}-\frac{k}{2}\right) L}-1\right.}\right]} \\
& \times i t_{\alpha \beta}\left(\sqrt{\sigma^{2}-\frac{3}{4} k^{2}}\right) \hat{g}_{(\gamma)}\left(k ; q_{i j}, q_{k}\right),
\end{aligned}
$$

$\alpha \neq \beta \neq \gamma$.

As discussed in the previous section, when the strength of potentials are not identical, Faddeev equations have no analytic solutions, and have to be solved numerically. Then solutions of $\hat{g}_{(\gamma)}$ amplitudes by solving Eq. (16) equations can be used as input to construct both free space and finite volume wave function according to Eqs. (18) and (19). Finally, the discrete spectra of three-body interaction in finite volume may be searched for numerically by using matching condition, Eq. (12).

\section{B. Exact solutions for equal strength $\delta$-function potentials: $V_{12}=V_{23}=V_{31}=V_{0}$}

In the case of equal strength of $\delta$-function potentials, $V_{12}=V_{23}=V_{31}=V_{0}$, the three-body interaction in finite volume is exactly solvable [83]. For totally symmetric incoming wave, see Eq. (13), the exact solutions of $g$ amplitude are

$$
\begin{aligned}
& g_{(1,2,3)}\left(k ; q_{i j}, q_{k}\right) \\
& =8 \pi \delta\left(k-q_{3}\right) i t\left(-q_{23}\right) \\
& +\frac{\left(1+\frac{\frac{i m V_{0}}{2}}{\sqrt{\sigma^{2}-\frac{3}{4} k^{2}}}\right) \frac{\left(-2 m V_{0}\right) 6 k}{\left(1+\frac{i m V_{0}}{2 q_{12}}\right)\left(1-\frac{i m V_{0}}{2 q_{23}}\right)\left(1-\frac{i m V_{0}}{2 q_{31}}\right)}}{\left(k-q_{3}-i \epsilon\right)\left(k-q_{2}-i \epsilon\right)\left(k-q_{1}+i \epsilon\right)},
\end{aligned}
$$

where $t(q)=-\frac{m V_{0}}{2 q+i m V_{0}}$ refers to two-body scattering amplitude. 
The totally symmetric wave function is expressed in terms of a single independent coefficient, see [83],

$$
\begin{aligned}
\psi^{\mathrm{sym}} & \left(r_{12}, r_{3} ; q_{i j}, q_{k}\right) \\
= & \left(A^{\mathrm{sym}}\left(r_{12}, r_{3}\right) e^{i q_{12} r_{12}}+A^{\mathrm{sym}}\left(-r_{12}, r_{3}\right) e^{-i q_{12} r_{12}}\right) e^{i q_{3} r_{3}} \\
& +\left(A^{\mathrm{sym}}\left(r_{31}, r_{2}\right) e^{i q_{23} r_{12}}+A^{\mathrm{sym}}\left(-r_{23}, r_{1}\right) e^{-i q_{23} r_{12}}\right) e^{i q_{1} r_{3}} \\
& +\left(A^{\mathrm{sym}}\left(r_{23}, r_{1}\right) e^{i q_{31} r_{12}}+A^{\mathrm{sym}}\left(-r_{31}, r_{2}\right) e^{-i q_{31} r_{12}}\right) e^{i q_{2} r_{3}},
\end{aligned}
$$

where $r_{23}=-\frac{r_{12}}{2}+r_{3}$ and $r_{31}=-\frac{r_{12}}{2}-r_{3}$, and

$$
\begin{aligned}
A^{\mathrm{sym}}\left(r_{12}, r_{3}\right)= & 1+\theta\left(r_{12}\right) 2 i t\left(q_{12}\right)\left[1+2 i t\left(-q_{23}\right)\right] \\
& +\theta\left(-r_{23}\right) 2 i t\left(-q_{23}\right)+\theta\left(-r_{31}\right) 2 i t\left(-q_{31}\right) \\
& -\theta\left(r_{12}\right) \theta\left(r_{23}\right) 4 i \mathbf{T}_{1}+\theta\left(r_{12}\right) \theta\left(-r_{31}\right) 4 i \mathbf{T}_{2},
\end{aligned}
$$

and

$$
\begin{aligned}
& i \mathbf{T}_{1}=\frac{\left(\frac{i m V_{0}}{2 q_{23}}\right)\left(\frac{i m V_{0}}{2 q_{31}}-\frac{i m V_{0}}{2 q_{12}}\right)}{\left(1+\frac{i m V_{0}}{2 q_{12}}\right)\left(1-\frac{i m V_{0}}{2 q_{23}}\right)\left(1-\frac{i m V_{0}}{2 q_{31}}\right)}, \\
& i \mathbf{T}_{2}=\frac{\left(\frac{i m V_{0}}{2 q_{31}}\right)\left(\frac{i m V_{0}}{2 q_{23}}-\frac{i m V_{0}}{2 q_{12}}\right)}{\left(1+\frac{i m V_{0}}{2 q_{12}}\right)\left(1-\frac{i m V_{0}}{2 q_{23}}\right)\left(1-\frac{i m V_{0}}{2 q_{31}}\right)} .
\end{aligned}
$$

We remark that the term $\psi_{(0)}^{\text {sym }}$ in Eq. (34) presented in [83] was a typo, see Eq. (21) for the correct version above. The totally symmetric finite volume wave function has the same structure as the free space wave function given in Eq. (21), the coefficient in finite volume is given by

$$
\begin{aligned}
A^{\mathrm{sym}(L)}\left(r_{12}, r_{3}\right)= & 4 i \mathbf{T}_{2}\left[\theta\left(r_{12}\right)+\frac{e^{i\left(\frac{2}{3} P+q_{2}\right) L}}{1-e^{i\left(\frac{2}{3} P+q_{2}\right) L}}\right] \\
& \times\left[\theta\left(-r_{31}\right)+\frac{e^{i\left(\frac{2}{3} P+q_{3}\right) L}}{1-e^{i\left(\frac{2}{3} P+q_{3}\right) L}}\right] \\
& -4 i \mathbf{T}_{1}\left[\theta\left(r_{12}\right)+\frac{e^{-i\left(\frac{(2}{3} P+q_{1}\right) L}}{1-e^{-i\left(\frac{2}{3} P+q_{1}\right) L}}\right] \\
& \times\left[\theta\left(r_{23}\right)+\frac{e^{i\left(\frac{2}{3} P+q_{3}\right) L}}{1-e^{i\left(\frac{2}{3} P+q_{3}\right) L}}\right] .
\end{aligned}
$$

As discussed in [83], the quantization conditions are obtained by considering the matching condition between free space wave function and finite volume wave function. For the case of equal strength $\delta$-function potentials, quantization conditions are given in simple forms,

$$
\begin{aligned}
\cot \left(\frac{P}{3}+\frac{q_{3}}{2}\right) L+\cot \left(\phi\left(-q_{31}\right)-\phi\left(-q_{23}\right)\right) & =0, \\
\cot \left(\frac{P}{3}+\frac{q_{1}}{2}\right) L+\cot \left(-\phi\left(-q_{31}\right)-\phi\left(q_{12}\right)\right) & =0, \\
\cot \left(\frac{P}{3}+\frac{q_{2}}{2}\right) L+\cot \left(\phi\left(-q_{23}\right)+\phi\left(q_{12}\right)\right) & =0,
\end{aligned}
$$

where two-body phase shift is given by $\phi(q)=$ $\cot ^{-1}\left(-\frac{2 q}{m V_{0}}\right)$.

\section{Strategy of searching allowed energy spectra}

The discrete energy spectra in finite volume are determined by the matching condition of wave functions in free space and finite volume, such as Eq. (12). Therefore, in principle, the task of obtaining three-body energy spectra in finite volume is to search all possible combination of $\left(q_{i j}, q_{k}\right)$, so that the matching condition, Eq. (12), is satisfied for an arbitrary $\left(r_{12}, r_{3}\right)$.

Normally, in order to explicitly remove $\left(r_{12}, r_{3}\right)$ dependence in matching condition, the quantization conditions may be further derived by expanding the wave functions in terms of certain orthogonal basis, see [84]. For example, the choice of basis may be made based on the asymptotic behavior of three-body wave functions [84], such as Bessel functions, $\left\{J_{J}(\sigma r), N_{J}(\sigma r)\right\}$, and $e^{i J \theta}$ in $\left(r_{12}, r_{3}\right)$ plane, where $(r, \theta)$ are the radius and polar angle of coordinate, $\left(r_{12}, r_{3}\right)$, respectively. Therefore, according to asymptotic behaviors of three-body wave function, the wave functions in free space and finite volume normally have the forms, see [84],

$$
\begin{aligned}
\psi\left(r_{12}, r_{3} ; q_{i j}, q_{k}\right)= & \sum_{J, J^{\prime}} e^{i J \theta}\left[c_{J, J^{\prime}}\left(q_{i j}, q_{k}\right) J_{J^{\prime}}(\sigma r)\right. \\
& \left.+\delta_{J, J^{\prime}} d_{J}\left(q_{i j}, q_{k}\right) N_{J^{\prime}}(\sigma r)\right], \\
\psi^{(L)}\left(r_{12}, r_{3} ; q_{i j}, q_{k}\right)= & \sum_{J, J^{\prime}} e^{i J \theta}\left[c_{J, J^{\prime}}^{(L)}\left(q_{i j}, q_{k}\right) J_{J^{\prime}}(\sigma r)\right. \\
& \left.+\delta_{J, J^{\prime}} d_{J}\left(q_{i j}, q_{k}\right) N_{J^{\prime}}(\sigma r)\right] .
\end{aligned}
$$

Hence, the matching condition, $\psi=\psi^{(L)}$, leads to the quantization conditions that are given by a determinant condition in terms of expansion coefficients alone,

$$
\operatorname{det}\left[c_{J, J^{\prime}}\left(q_{i j}, q_{k}\right)-c_{J, J^{\prime}}^{(L)}\left(q_{i j}, q_{k}\right)\right]=0 .
$$

With the exception of a few special cases, such as, equal strength $\delta$-function potentials, the quantization conditions usually do not possess a simple analytic expression and appear to be messy and complicated. Moreover, the expansion must be truncated in practice to solve a determinant condition, thus, the convergence of expansion somehow more or less depends on the choice of expansion 
basis of wave functions. Therefore, instead of making efforts to obtain quantization conditions and solve the determinant condition, such as in Eq. (28), we propose to solve the matching condition directly. Though, the explicit expression of quantization conditions is sacrificed, the procedure and strategy of obtaining discrete energy spectra is clearer and more straightforward. First, the Faddeev equations that define the dynamics of three-body interaction, such as in Eq. (5), are solved numerically. Next, the solutions of Faddeev equations are used as input to construct both free space and finite volume wave functions, see Eqs. (2) and (11). At last, the discrete energy spectra determined by periodic lattice structures are obtained by searching for the matching condition, $\psi=\psi^{(L)}$, directly.

In this subsection, using scattering of three-boson at the limit of equal strength $\delta$-function potentials as a numerical test, we solve Faddeev equations presented in subsection III A, and use the solution of $g$-amplitude as input to construct wave functions and further seek the discrete spectra that satisfy the matching condition in Eq. (12). The numerical results are compared with the exact solutions. The comparison of the numerical solutions of $g$-amplitudes, free space wave function and finite volume wave function with exact solutions are presented in Fig. 1-3 respectively. The matching condition, $\psi=\psi^{(L)}$, is solved numerically by the root finding method, more specifically, a function, $\mathcal{M}\left(q_{12}, q_{3}\right)$, is introduced,

$$
\begin{aligned}
& \mathcal{M}\left(q_{12}, q_{3}\right) \\
& =\frac{1}{N} \sum_{\left(r_{12}, r_{3}\right)=1}^{N} \frac{\left|\psi\left(r_{12}, r_{3} ; q_{12}, q_{3}\right)-\psi^{(L)}\left(r_{12}, r_{3} ; q_{12}, q_{3}\right)\right|}{\left|\psi\left(r_{12}, r_{3} ; q_{12}, q_{3}\right)\right|},
\end{aligned}
$$

where sum of $\left(r_{12}, r_{3}\right)$ are carried out by choosing some discrete values. For example, in present work, $\left(r_{12}, r_{3}\right)$ space is discretized in terms of polar coordinate, $(r, \theta)$. About 30 points of $r$ values in range $r \in[1,5]$ and 100 points of $\theta \in[0,2 \pi]$ are taken in the sum. $N$ refers to the total number of discrete points of $\left(r_{12}, r_{3}\right)$ in the sum. For nontrivial solutions of three-body energy spectra (none of particle momentum coincides with $\frac{2 \pi}{L} n, n \in \mathcal{Z}$ ), the possible discrete values of pair $\left(q_{12}, q_{3}\right)$ are searched for by performing a root finding of condition, $\mathcal{M}\left(q_{12}, q_{3}\right)=0$. The results are compared with the solutions given by quantization condition in Eq. (25), and presented in Fig. 4. We remark that the solutions of quantization condition for $q_{i j}=0$ are excluded in Fig. 4 . It is not difficult to see that the wave functions vanish due to the symmetry of three-body for $q_{i j}=0$ at the limit of $V_{12}=V_{23}=V_{31}=V_{0}$. Thus, the solutions for $q_{i j}=0$ are considered trivial and are not included in Fig. 4.

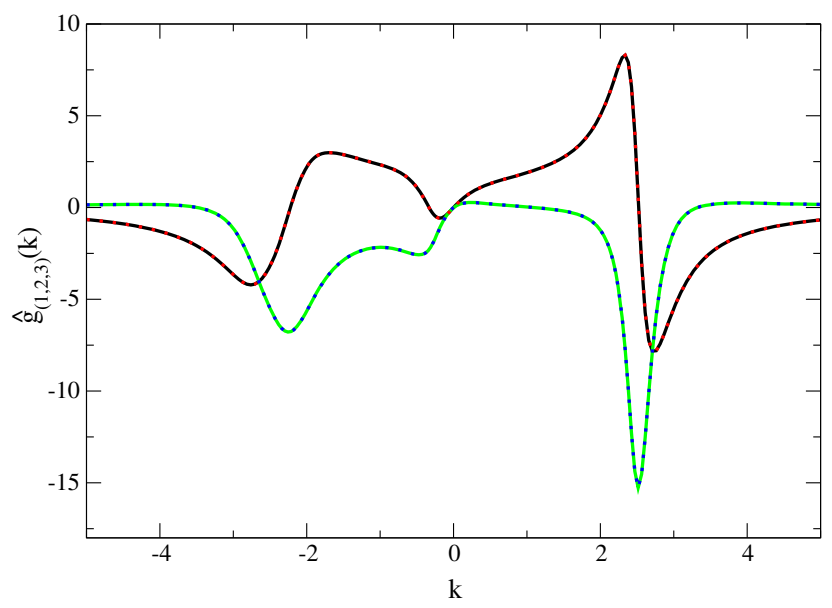

FIG. 1. The comparison of numerical solutions of integral Eq. (16), $\hat{g}_{(1,2,3)}$, with exact solutions given in Eq. (20). Solid black and solid green curves represent real and imaginary parts of numerical solutions, and dotted red and blue are real and imaginary parts of exact solutions respectively. The parameters of the toy model are chosen as $m V_{0}=2.0, q_{12}=1.0+0.4 i$ and $q_{3}=2.5+0.2 i$, where an imaginary part is given to both $\left(q_{12}, q_{3}\right)$ to smooth out the curves near the pole positions only for a better visualization purposes.
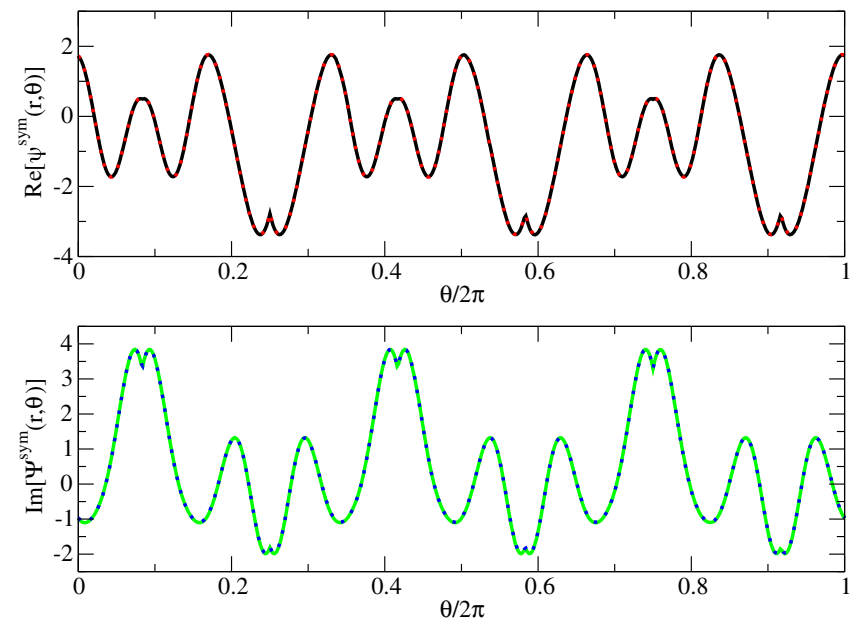

FIG. 2. The comparison of numerical solution of free space wave function, $\psi^{\mathrm{sym}}$, constructed by using the solution of Faddeev equations and Eq. (18) with exact solution given in Eqs. (21)-(22). The real and imaginary parts of free space wave function are presented in upper and lower panels respectively. Solid black and dotted red curves in the upper panel represent real part of numerical solution and exact solution respectively, and solid green and dotted blue curves in the lower panel represent the imaginary part of numerical solution and exact solution respectively. The parameters are chosen as $m V_{0}=2.0, q_{12}=1.0$, and $q_{3}=2.5$. The wave function as function of $(r, \theta)$ is plotted with a fixed $r=5.5$. 

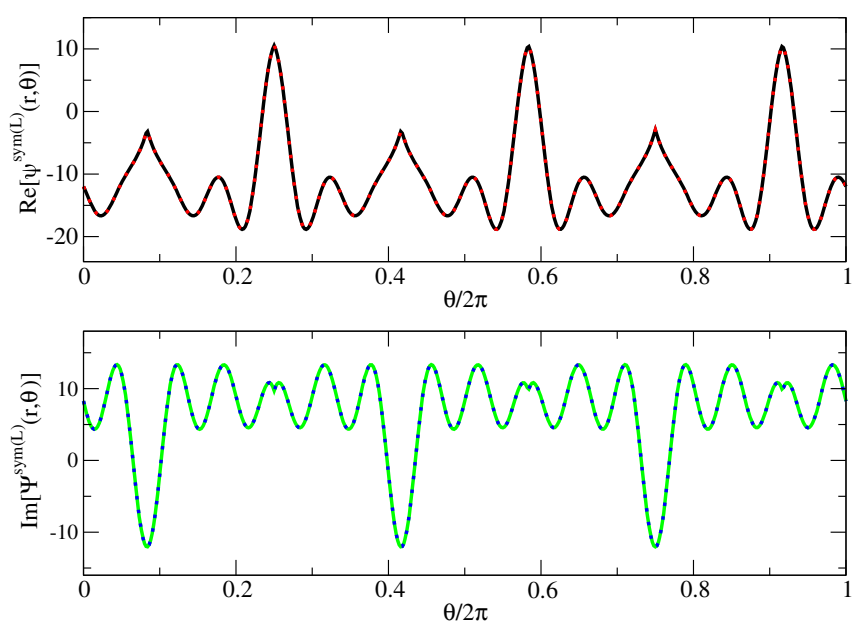

FIG. 3. The comparison of numerical solutions of center of mass $(P=0)$ finite volume wave function, $\psi^{\operatorname{sym}(L)}$, constructed by using the solution of Faddeev equations and Eq. (19) with exact solutions given by Eq. (24). The real and imaginary parts of finite volume wave function are presented in the upper and lower panels respectively. The color coding, line styles and parameters are the same as Fig. 2. The finite volume wave function as function of $(r, \theta)$ is plotted with a fixed $r=5.5$.

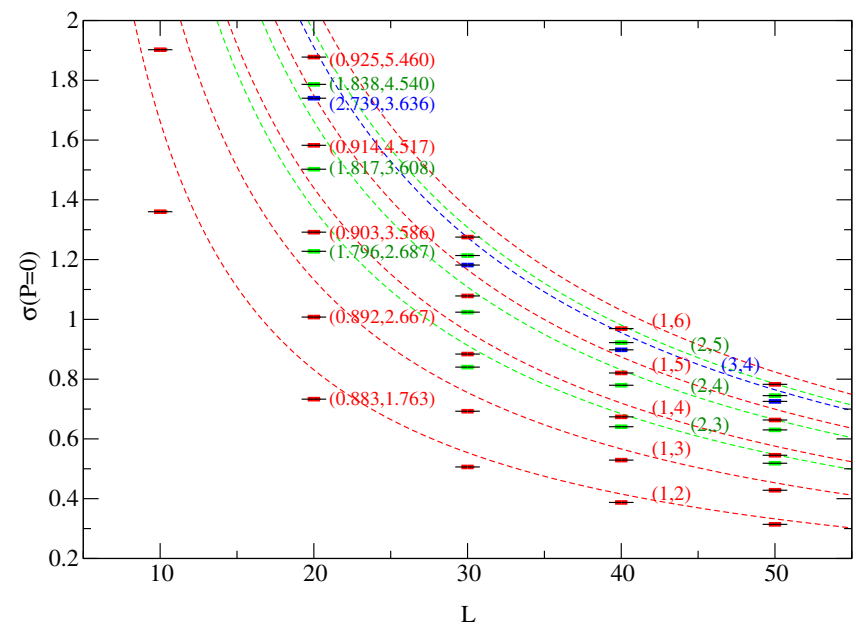

FIG. 4. The center of mass $(P=0)$ three-body energy spectra, $\sigma=\sqrt{m E}=\sqrt{q_{12}^{2}+\frac{3}{4} q_{3}^{2}}$, as function of lattice size, $L$. The solid black bars and colored boxes represent solutions of the secular equation, Eq. (25), and matching condition by solving $\mathcal{M}\left(q_{12}, q_{3}\right)=0$ respectively. The red, green and blue dashed curves represent the noninteracting energy spectra: $\frac{2 \pi}{L} \sqrt{\left(\frac{n_{1}-n_{2}}{2}\right)^{2}+\frac{3}{4}\left(n_{1}+n_{2}\right)^{2}}$ with $\left(n_{1}, n_{2}\right) \in \mathbb{Z}$. The colored lines are labeled by pairs of integers, $\left(n_{1}, n_{2}\right)$, which are associated to momenta of noninteracting particles by relations: $p_{1,2,3}=\frac{2 \pi}{L} n_{1,2,3}$. As examples of both discrete energy and momenta in a finite box, the three-body energy levels for $L=$ 20 are also labeled by pairs of discrete values of $\left(q_{12}, q_{3}\right)$ next to their corresponding energy level.

\section{DISCUSSION AND CONCLUSION}

As mentioned in previous sections, the dynamics of three-body interaction are completely determined by Faddeev equations regardless of the three-particle interacting in free space or finite box. When the periodic boundary condition is considered, the allowed energy spectra are constrained by the matching condition between free space three-body wave function and finite volume wave function, eventually becoming discrete. Therefore, seeking discrete three-body energy spectra in a finite box can be carried out by two independent procedures. The first requires solving dynamical Faddeev equations and using the solutions of Faddeev equations to construct wave functions. As for the second, instead of seeking an analytic expression of quantization conditions for finite volume three-body interaction that may be derived from matching condition of three-body wave functions, we propose to search for discrete energy spectra by using the matching condition directly.

In this work, we demonstrated this approach by considering the scattering of three spinless bosons interacting with $\delta$-function potentials. In this case, the exact analytic solutions exist at the limit of equal strength of $\delta$-function potential among all pairs. The finite volume three-body interaction in this toy model is then solved numerically, and the discrete energy spectra are searched for by using the matching condition of wave functions. Finally, all the numerical results are compared with exact solutions presented in Sec. III. We want to stress that although a specific toy model with only pairwise interaction is solved in this work, this approach is in fact not limited to only pair-wise interaction. The strategy and procedure is applicable to more general cases when three-body forces are involved, due to the fact that the three-body dynamics and constraints on allowed energy spectra by periodic lattice structure are two independent procedures and can be carried out separately. Lastly, we would like to remark that as demonstrated in this work, though the configurational space wave function approach seems work perfectly well in low dimensional space, the extension of the wave function approach into real world in three spatial dimensions still needs to be examined and studied with care. Especially, the connection of wave function approach in configuration space to the approaches developed by other groups [73-80,85-88] is still unclear and deserves a closer look. These issues will be addressed in our future works.

\section{ACKNOWLEDGMENTS}

We acknowledge support from the Department of Physics and Engineering, California State University, Bakersfield, CA. We also thank Tyler Morris for carefully reading through this manuscript. 


\section{APPENDIX A: THREE-BODY INTERACTION AND FADDEEV EQUATIONS}

In this section, we consider the scattering of three spinless particles of equal masses, interacting by $\delta$-function potentials of strength, $V_{\alpha \beta}$, between $\alpha$ th and $\beta$ th particles. The three-particle wave function satisfies Schrödinger equation,

$$
\begin{aligned}
& {\left[-\frac{1}{2 m} \sum_{i=1}^{3} \frac{d^{2}}{d x_{i}^{2}}+V_{12} \delta\left(r_{12}\right)+V_{23} \delta\left(r_{23}\right)+V_{31} \delta\left(r_{31}\right)-E\right]} \\
& \times \Psi\left(x_{1}, x_{2}, x_{3} ; p_{1}, p_{2}, p_{3}\right)=0,
\end{aligned}
$$

where $m, p_{i}(i=1,2,3)$ and $E=\sum_{i=1}^{3} \frac{p_{i}^{2}}{2 m}$ refer to the mass of particle, the particle's initial momenta and three-body total energy respectively. As shown in [83], the center of mass, relative positions and corresponding conjugate momenta among particles are defined by $R=\frac{x_{1}+x_{2}+x_{3}}{3}$, $r_{i j}=x_{i}-x_{j}$ and $r_{k}=\frac{x_{i}+x_{j}}{2}-x_{k}, P=p_{1}+p_{2}+p_{3}, q_{i j}=$ $\frac{p_{i}-p_{j}}{2}$ and $q_{k}=\frac{p_{i}+p_{j}-2 p_{k}}{3}(i \neq j \neq k)$ respectively. Because of translational invariance, the center of mass motion is described by a plane wave. The total three particles wave function is given by, $\Psi\left(x_{1}, x_{2}, x_{3} ; p_{1}, p_{2}, p_{3}\right)=$ $e^{i P R} \psi\left(r_{\alpha \beta}, r_{\gamma} ; q_{i j}, q_{k}\right)$, where $\psi\left(r_{\alpha \beta}, r_{\gamma} ; q_{i j}, q_{k}\right)$ describes relative motions of three particles.

\section{Scattering of three free particles}

With an incoming wave of three free particles state, $\Psi_{(0)}$, three-body wave function has the form $[12,13]$, $\Psi=\Psi_{(0)}+\sum_{\gamma=1}^{3} \Psi_{(\gamma)}$, where $\Psi_{(\gamma)}$ satisfies equation,

$$
\begin{aligned}
& {\left[-\frac{1}{2 m} \sum_{i=1}^{3} \frac{d^{2}}{d x_{i}^{2}}+V_{\alpha \beta} \delta\left(r_{\alpha \beta}\right)-E\right] \Psi_{(\gamma)}} \\
& \quad=-V_{\alpha \beta} \delta\left(r_{\alpha \beta}\right)\left[\Psi_{(0)}+\Psi_{(\alpha)}+\Psi_{(\beta)}\right], \quad \gamma \neq \alpha \neq \beta .
\end{aligned}
$$

As shown in [83], the Lippmann-Schwinger equation for relative wave function, $\psi_{(\gamma)}$, can be obtained as

$$
\begin{aligned}
\psi_{(\gamma)}\left(r_{\alpha \beta}, r_{\gamma} ; q_{i j}, q_{k}\right) & \\
= & \int_{-\infty}^{\infty} \frac{d k}{2 \pi} e^{i \sqrt{\sigma^{2}-\frac{3}{4} k^{2}}\left|r_{\alpha \beta}\right|} e^{i k r_{\gamma}} i t_{\alpha \beta}\left(\sqrt{\sigma^{2}-\frac{3}{4} k^{2}}\right) \\
& \times \int_{-\infty}^{\infty} d r_{\alpha \beta}^{\prime} d r_{\gamma}^{\prime} e^{-i k r_{\gamma}^{\prime}} \delta\left(r_{\alpha \beta}^{\prime}\right)\left[\psi_{(0)}\left(r_{\alpha \beta}^{\prime}, r_{\gamma}^{\prime} ; q_{i j}, q_{k}\right)\right. \\
& \left.+\psi_{(\alpha)}\left(r_{\beta \gamma}^{\prime}, r_{\alpha}^{\prime} ; q_{i j}, q_{k}\right)+\psi_{(\beta)}\left(r_{\gamma \alpha}^{\prime}, r_{\beta}^{\prime} ; q_{i j}, q_{k}\right)\right], \\
\alpha & \neq \beta \neq \gamma,
\end{aligned}
$$

where $t_{\alpha \beta}(k)=-\frac{m V_{\alpha \beta}}{2 k+i m V_{\alpha \beta}}$ refers to the two-body scattering amplitude between $\alpha$ th and $\beta$ th particles for a $\delta$-function potential interaction, and $\sigma^{2}=m E-\frac{P^{2}}{6}=q_{i j}^{2}+\frac{3}{4} q_{k}^{2}$.

Instead of solving Faddeev $T$-matrix equations, see [83], numerically, it is more convenient to introduce amplitudes, $g_{(\gamma)}$, by

$$
\begin{aligned}
g_{(\gamma)}\left(k ; q_{i j}, q_{k}\right) \\
=\int_{-\infty}^{\infty} d r e^{-i k r}\left[\psi_{(\alpha)}\left(r,-\frac{r}{2} ; q_{i j}, q_{k}\right)\right. \\
\left.\quad+\psi_{(\beta)}\left(r,-\frac{r}{2} ; q_{i j}, q_{k}\right)\right] .
\end{aligned}
$$

The relative wave function, $\psi_{(\gamma)}$, thus can be written as

$$
\begin{aligned}
\psi_{(\gamma)}\left(r_{\alpha \beta}, r_{\gamma} ; q_{i j}, q_{k}\right) \\
=\int_{-\infty}^{\infty} \frac{d k}{2 \pi} e^{i \sqrt{\sigma^{2}-\frac{3}{4} k^{2}}\left|r_{\alpha \beta}\right|} e^{i k r_{\gamma}} i t_{\alpha \beta}\left(\sqrt{\sigma^{2}-\frac{3}{4} k^{2}}\right) \\
\quad \times\left[\int_{-\infty}^{\infty} d r_{\gamma}^{\prime} e^{-i k r_{\gamma}^{\prime}} \psi_{(0)}\left(0, r_{\gamma}^{\prime} ; q_{i j}, q_{k}\right)+g_{(\gamma)}\left(k ; q_{i j}, q_{k}\right)\right] .
\end{aligned}
$$

Using Eqs. (A4) and (A5), the integral equations for $g_{(\gamma)}$-amplitude are obtained,

$$
\begin{aligned}
g_{(\gamma)}( & \left.; q_{i j}, q_{k}\right) \\
= & g_{(\gamma)}^{(0)}\left(k ; q_{i j}, q_{k}\right)+i \int_{-\infty}^{\infty} \frac{d q}{2 \pi} \frac{2 \sqrt{\sigma^{2}-\frac{3}{4} q^{2}}}{\left(\sigma^{2}-\frac{3}{4} q^{2}\right)-\left(k+\frac{q}{2}\right)^{2}+i \epsilon} \\
& \times\left[i t_{\beta \gamma}\left(\sqrt{\sigma^{2}-\frac{3}{4} q^{2}}\right) g_{(\alpha)}\left(q ; q_{i j}, q_{k}\right)\right. \\
& \left.+i t_{\gamma \alpha}\left(\sqrt{\sigma^{2}-\frac{3}{4} q^{2}}\right) g_{(\beta)}\left(q ; q_{i j}, q_{k}\right)\right],
\end{aligned}
$$

where $g_{(\gamma)}^{(0)}$ is given by,

$$
\begin{aligned}
g_{(\gamma)}^{(0)} & \left(k ; q_{i j}, q_{k}\right) \\
= & i \int_{-\infty}^{\infty} \frac{d q}{2 \pi} \frac{2 \sqrt{\sigma^{2}-\frac{3}{4} q^{2}}}{\left(\sigma^{2}-\frac{3}{4} q^{2}\right)-\left(k+\frac{q}{2}\right)^{2}+i \epsilon} \\
& \times\left[i t_{\beta \gamma}\left(\sqrt{\sigma^{2}-\frac{3}{4} q^{2}}\right) \int_{-\infty}^{\infty} d r_{\alpha} e^{-i q r_{\alpha}} \psi_{(0)}\left(0, r_{\alpha} ; q_{i j}, q_{k}\right)\right. \\
& \left.+i t_{\gamma \alpha}\left(\sqrt{\sigma^{2}-\frac{3}{4} q^{2}}\right) \int_{-\infty}^{\infty} d r_{\beta} e^{-i q r_{\beta}} \psi_{(0)}\left(0, r_{\beta} ; q_{i j}, q_{k}\right)\right] .
\end{aligned}
$$


The $g_{(\gamma)}$-amplitude is related to standard Faddeev $T$-amplitude by

$$
\begin{aligned}
T_{(\gamma)}\left(k ; q_{i j}, q_{k}\right)= & 2 \sqrt{\sigma^{2}-\frac{3}{4} k^{2}} t_{\alpha \beta}\left(\sqrt{\sigma^{2}-\frac{3}{4} k^{2}}\right) \\
& \times\left[\int_{-\infty}^{\infty} d r_{\gamma} e^{-i k r_{\gamma}} \psi_{(0)}\left(0, r_{\gamma} ; q_{i j}, q_{k}\right)\right. \\
& \left.+g_{(\gamma)}\left(k ; q_{i j}, q_{k}\right)\right],
\end{aligned}
$$

the full three-body scattering amplitude is given by

$$
T\left(k_{\alpha \beta}, k_{\gamma} ; q_{i j}, q_{k}\right)=\sum_{\delta=1}^{3} T_{(\delta)}\left(k_{\delta} ; q_{i j}, q_{k}\right),
$$

where $k_{\alpha}=-k_{\alpha \beta}-\frac{k_{y}}{2}$ and $k_{\beta}=k_{\alpha \beta}-\frac{k_{y}}{2}$.

\section{Scattering on a two-body bound state}

For the case of scattering on a bound state, e.g., ith particle incident on a bound state of $(j k)$ pair, three-body wave function thus has the form of $\Psi=\sum_{\gamma=1}^{3} \Psi_{(\gamma)}$, and the Lippmann-Schwinger equation for relative wave function reads,

$$
\begin{aligned}
\psi_{(\gamma)}\left(r_{\alpha \beta}, r_{\gamma} ; q_{i j}, q_{k}\right)= & \delta_{\gamma, i} \phi_{(\gamma)}^{B}\left(r_{\alpha \beta}\right) e^{i q_{i}^{B} r_{\gamma}} \\
& +\int_{-\infty}^{\infty} \frac{d k}{2 \pi} e^{i \sqrt{\sigma^{2}-\frac{3}{4} k^{2}}\left|r_{\alpha \beta}\right|} e^{i k r_{\gamma}} \\
& \times i t_{\alpha \beta}\left(\sqrt{\sigma^{2}-\frac{3}{4} k^{2}}\right) g_{(\gamma)}\left(k ; q_{i j}, q_{k}\right),
\end{aligned}
$$

where the bound state wave function in pair $(j k)$ is given by $\phi_{(\gamma)}^{B}\left(r_{\alpha \beta}\right)=\sqrt{-\frac{m V_{j k}}{2}} e^{\frac{m V_{j k}}{2} \mid} r_{\alpha \beta} \mid$, and $q_{i}^{B}=\sqrt{\sigma^{2}+\frac{3}{4}\left(\frac{m V_{j k}}{2}\right)^{2}}$ refers to the relative momentum between incident ith particle and pair $(j k)$ bound state. The integral equations for $g_{(\gamma)}$-amplitude for scattering on a bound state also has the same form of Eq. (A6), where for the case of scattering on a bound state in $(j k)$ pair, $g_{(\gamma)}^{(0)}$ is given by

$$
\begin{aligned}
g_{(\gamma)}^{(0)}\left(k ; q_{i j}, q_{k}\right)= & \delta_{\alpha, i} \int d r_{\alpha} \phi_{(\alpha)}^{B}\left(r_{\alpha}\right) e^{-i\left(k+\frac{q_{i}^{B}}{2}\right) r_{\alpha}} \\
& +\delta_{\beta, i} \int d r_{\beta} \phi_{(\beta)}^{B}\left(r_{\beta}\right) e^{-i\left(k+\frac{q_{i}^{B}}{2}\right) r_{\beta}} .
\end{aligned}
$$

\section{Exact solutions for equal strength of $\delta$-function potentials: $V_{12}=V_{23}=V_{31}=V_{0}$}

Only for the special case with equal strength of $\delta$-potential among all pairs, $V_{12}=V_{23}=V_{31}=V_{0}$, three-body interactions are in fact exactly solvable $[83,91,92]$. The exact solutions can be used for testing and verifying the numerical approach, and also for completeness, the exact solutions of Faddeev equations, Eq. (A6), for scattering of three free particles with incoming wave $e^{i q_{12} r_{12}} e^{i q_{3} r_{3}}$ and for scattering on a bound state of pair (12) respectively are presented in following.

\section{a. Scattering of three free particles with incoming wave $e^{i q_{12} r_{12}} e^{i q_{3} r_{3}}$}

For incoming wave $e^{i q_{12} r_{12}} e^{i q_{3} r_{3}}, g_{(\gamma)}^{(0)}$ is given by

$$
\begin{aligned}
g_{(3)}^{(0)}\left(k ; q_{i j}, q_{k}\right)= & i \frac{2 q_{23} i t\left(-q_{23}\right)}{\left(k-q_{3}-i \epsilon\right)\left(k-q_{2}-i \epsilon\right)} \\
& +i \frac{2 q_{31} i t\left(-q_{31}\right)}{\left(k-q_{3}-i \epsilon\right)\left(k-q_{1}+i \epsilon\right)} \\
& +i t\left(-q_{23}\right) 2 \pi \delta\left(k-q_{3}\right),
\end{aligned}
$$

$$
\begin{aligned}
g_{(1)}^{(0)}\left(k ; q_{i j}, q_{k}\right)= & i \frac{2 q_{31} i t\left(-q_{31}\right)}{\left(k-q_{3}-i \epsilon\right)\left(k-q_{1}+i \epsilon\right)} \\
& -i \frac{2 q_{12} i t\left(q_{12}\right)}{\left(k-q_{2}-i \epsilon\right)\left(k-q_{1}+i \epsilon\right)},
\end{aligned}
$$

$$
\begin{aligned}
g_{(2)}^{(0)}\left(k ; q_{i j}, q_{k}\right)= & -i \frac{2 q_{12} i t\left(q_{12}\right)}{\left(k-q_{2}-i \epsilon\right)\left(k-q_{1}+i \epsilon\right)} \\
& +i \frac{2 q_{23} i t\left(-q_{23}\right)}{\left(k-q_{3}-i \epsilon\right)\left(k-q_{2}-i \epsilon\right)} \\
& +i t\left(-q_{23}\right) 2 \pi \delta\left(k-q_{3}\right),
\end{aligned}
$$

where $t(q)=-\frac{m V_{0}}{2 q+i m V_{0}}$. The exact solutions of $g$-amplitude are

$$
\begin{aligned}
& g_{(3)}\left(k ; q_{i j}, q_{k}\right)=i t\left(-q_{23}\right) 2 \pi \delta\left(k-q_{3}\right) \\
& +\frac{\left(1+\frac{\frac{i m V_{0}}{2}}{\sqrt{\sigma^{2}-\frac{3}{4} k^{2}}}\right) \frac{\left(-2 m V_{0}\right)\left(k+\frac{q_{3}}{2}\right)}{\left(1+\frac{i m V_{0}}{2 q_{12}}\right)\left(1-\frac{i m V_{0}}{2 q_{23}}\right)\left(1-\frac{i m V_{0}}{2 q_{31}}\right)}}{\left(k-q_{3}-i \epsilon\right)\left(k-q_{2}-i \epsilon\right)\left(k-q_{1}+i \epsilon\right)}, \\
& g_{(1)}\left(k ; q_{i j}, q_{k}\right)=\frac{\left(1+\frac{\frac{i m V_{0}}{2}}{\sqrt{\sigma^{2}-\frac{3}{4} k^{2}}}\right) \frac{\left(-2 m V_{0}\right)\left(k-\frac{q_{12}}{2}-\frac{q_{3}}{4}-\frac{i m V_{0}}{2}\right)}{\left(1+\frac{i m V_{0}}{2 q_{12}}\right)\left(1-\frac{i m V_{0}}{22 q_{2}}\right)\left(1-\frac{i m V_{0}}{2 q_{31}}\right)}}{\left(k-q_{3}-i \epsilon\right)\left(k-q_{2}-i \epsilon\right)\left(k-q_{1}+i \epsilon\right)}, \\
& g_{(2)}\left(k ; q_{i j}, q_{k}\right)=i t\left(-q_{23}\right) 2 \pi \delta\left(k-q_{3}\right) \\
& +\frac{\left(1+\frac{\frac{i m V_{0}}{2}}{\sqrt{\sigma^{2}-\frac{3}{4} k^{2}}}\right) \frac{\left(-2 m V_{0}\right)\left(k+\frac{q_{12}}{2}-\frac{q_{3}}{4}+\frac{i m V_{0}}{4}\right)}{\left(1+\frac{i m V_{12}}{2 q_{12}}\right)\left(1-\frac{i m V_{0}}{2 q_{23}}\right)\left(1-\frac{i m V_{0}}{2 q_{31}}\right)}}{\left(k-q_{3}-i \epsilon\right)\left(k-q_{2}-i \epsilon\right)\left(k-q_{1}+i \epsilon\right)} .
\end{aligned}
$$




\section{b. Scattering on a bound state of pair (12)}

For incoming wave $\phi_{(3)}^{B}\left(r_{12}\right) e^{i q_{3}^{B} r_{3}}$, the $g_{(\gamma)}^{(0)}$ is given by

$$
\begin{aligned}
g_{(3)}^{(0)}\left(k ; q_{i j}, q_{k}\right) & =0, \\
g_{(1,2)}^{(0)}\left(k ; q_{i j}, q_{k}\right) & =\frac{2 \sqrt{\left(\frac{m V_{0}}{2}\right)^{2}} \sqrt{-\frac{m V_{0}}{2}}}{\left(k+\frac{q_{3}^{B}}{2}\right)^{2}+\left(\frac{m V_{0}}{2}\right)^{2}} .
\end{aligned}
$$

The exact solutions of $g$-amplitude are

$$
\begin{aligned}
g_{(3)}\left(k ; q_{i j}, q_{k}\right)= & \frac{4}{3} \sqrt{\left(\frac{m V_{0}}{2}\right)^{2}} \sqrt{-\frac{m V_{0}}{2}} \\
& \times \frac{\left(1+\frac{\frac{i m V_{0}}{2}}{\sqrt{\sigma^{2}-\frac{3}{4} k^{2}}}\right) \frac{\left(\frac{3 q_{3}}{q_{3}}-\frac{i m V_{0}}{2}+\frac{i m V_{0}}{2}\right)}{\left(k-q_{3}-i \epsilon\right)\left(k-q_{2}-i \epsilon\right)\left(k-q_{1}+i \epsilon\right)},}{\text { ( } \left.)+\frac{q_{3}}{2}+\frac{i m V_{0}}{2}\right)}
\end{aligned}
$$

$$
\begin{aligned}
g_{(1,2)}\left(k ; q_{i j}, q_{k}\right)= & \frac{4}{3} \sqrt{\left(\frac{m V_{0}}{2}\right)^{2}} \sqrt{-\frac{m V_{0}}{2}} \\
& \times \frac{\left(1+\frac{\frac{i m V_{0}}{2}}{\sqrt{\sigma^{2}-\frac{3}{4} k^{2}}}\right) \frac{\left(\frac{3 q_{3}}{2}-\frac{i m V_{0}}{2}\right)}{\left(\frac{q_{3}}{2}+\frac{i m V_{0}}{2}\right)}\left(k-\frac{q_{3}}{4}-\frac{i m V_{0}}{4}\right)}{\left(k-q_{3}-i \epsilon\right)\left(k-q_{2}-i \epsilon\right)\left(k-q_{1}+i \epsilon\right)} .
\end{aligned}
$$

\section{Numerical test for scattering of three free particles with an incoming wave $e^{i q_{12} r_{12}} e^{i q_{3} r_{3}}$}

In general, Faddeev equations, Eq. (A6), have to be solved numerically. The numerical approach is rather straightforward for the case of scattering on a bound state, the expression of $g_{(\gamma)}^{(0)}$ [see Eq. (A18)] does not contain $\delta$-function type singularities. Thus, Eq. (A6) is standard Fredholm-type integral equation, and can be solved easily by the matrix inversion method. Special care has to be given to the case of the scattering of three free particles. In this case, $g_{(\gamma)}^{(0)}$ [see Eqs. (A12)-(A14)] does indeed contain $\delta$-function type singularities. The singularities in Eq. (A6) can be removed by redefining $g_{(\gamma)}$ 's. For example, given that $g_{(3)}^{(0)}$ contains singular term $i t_{23}\left(-q_{23}\right) 2 \pi \delta\left(k-q_{3}\right)$, by a shifting in $g_{(3)}$,

$g_{(3)}\left(k ; q_{i j}, q_{k}\right)=\hat{g}_{(3)}\left(k ; q_{i j}, q_{k}\right)+i t_{23}\left(-q_{23}\right) 2 \pi \delta\left(k-q_{3}\right)$,

the new integral equations for $\hat{g}_{(\gamma)}$ 's are free of $\delta$-function type singularities, and are also Fredholm-type equations. In addition, extra care must be taken when it comes to the branch cut of square root terms, and pole contributions in Faddeev equations. The pole contributions are handled by using standard $i \epsilon$ prescription, see Eq. (A6) for instance. As for branch cut contribution, we adopt the same convention as used in [83], for the square root terms, $\sqrt{q_{\alpha \beta}^{2}}$, we assign a small imaginary part to $q_{12} \rightarrow q_{12}+i 0^{+}$, the imaginary part for $q_{23} \rightarrow q_{23}-i 0^{+}$and $q_{31} \rightarrow q_{31}-i 0^{+}$are thus determined by relations, $q_{23}=-\frac{1}{2} q_{12}+\frac{3}{4} q_{3}$ and $q_{31}=$ $-\frac{1}{2} q_{12}-\frac{3}{4} q_{3}$ respectively. In addition, our convention for complex square root is given by $\sqrt{q^{2} \pm i 0^{+}}= \pm \sqrt{q^{2}}$. Therefore, $\sqrt{\left(q_{12}+i 0^{+}\right)^{2}}=q_{12}, \sqrt{\left(q_{23}-i 0^{+}\right)^{2}}=-q_{23}$ and $\sqrt{\left(q_{31}-i 0^{+}\right)^{2}}=-q_{31}$.

As demonstrations of some numerical tests, the $g_{(\gamma)}$ 's equations are solved numerically for an incoming wave of $e^{i q_{12} r_{12}} e^{i q_{3} r_{3}}$ and compared with the exact solutions presented in Sec. A 3 a. As mentioned previously, the $\delta$ function type singularities must be removed by shifting $g_{(\gamma)}$ 's,

$$
\begin{aligned}
g_{(1)}\left(k ; q_{i j}, q_{k}\right) & =\hat{g}_{(1)}\left(k ; q_{i j}, q_{k}\right), \\
g_{(3,2)}\left(k ; q_{i j}, q_{k}\right) & =\hat{g}_{(3,2)}\left(k ; q_{i j}, q_{k}\right)+i t_{23}\left(-q_{23}\right) 2 \pi \delta\left(k-q_{3}\right) .
\end{aligned}
$$

We thus obtain integral equations for $\hat{g}_{(\gamma)}$ 's,

$$
\begin{aligned}
\hat{G}(k)= & \hat{G}^{(0)}(k)+i \int_{-\infty}^{\infty} \frac{d q}{2 \pi} \frac{2 \sqrt{\sigma^{2}-\frac{3}{4} q^{2}}}{\sigma^{2}-\frac{3}{4} q^{2}-\left(k+\frac{q}{2}\right)^{2}+i \epsilon} \\
& \times \mathcal{K}\left(\sqrt{\sigma^{2}-\frac{3}{4} q^{2}}\right) \hat{G}(q),
\end{aligned}
$$

where $\hat{G}$ and $\hat{G}^{(0)}$ stand for column vectors $\left(\hat{g}_{(3)}, \hat{g}_{(1)}, \hat{g}_{(2)}\right)^{T}$ and $\left(\hat{g}_{(3)}^{(0)}, \hat{g}_{(1)}^{(0)}, \hat{g}_{(2)}^{(0)}\right)^{T}$ respectively. The dependence on initial momenta $\left(q_{i j}, q_{k}\right)$ are dropped in the equation above. The matrix $\mathcal{K}$ is given by

$$
\mathcal{K}(q)=\left[\begin{array}{ccc}
0 & i t_{23}(q) & i t_{31}(q) \\
i t_{12}(q) & 0 & i t_{31}(q) \\
i t_{12}(q) & i t_{23}(q) & 0
\end{array}\right],
$$

and

$$
\begin{aligned}
\hat{g}_{(3)}^{(0)}\left(k ; q_{i j}, q_{k}\right)= & i \frac{2 q_{23} i t_{23}\left(-q_{23}\right)}{\left(k-q_{3}-i \epsilon\right)\left(k-q_{2}-i \epsilon\right)} \\
& +i \frac{2 q_{31} i t_{31}\left(-q_{31}\right)}{\left(k-q_{3}-i \epsilon\right)\left(k-q_{1}+i \epsilon\right)} \\
& -i \frac{2 q_{12} i t_{31}\left(q_{12}\right) i t_{23}\left(-q_{23}\right)}{\left(k-q_{2}-i \epsilon\right)\left(k-q_{1}+i \epsilon\right)},
\end{aligned}
$$



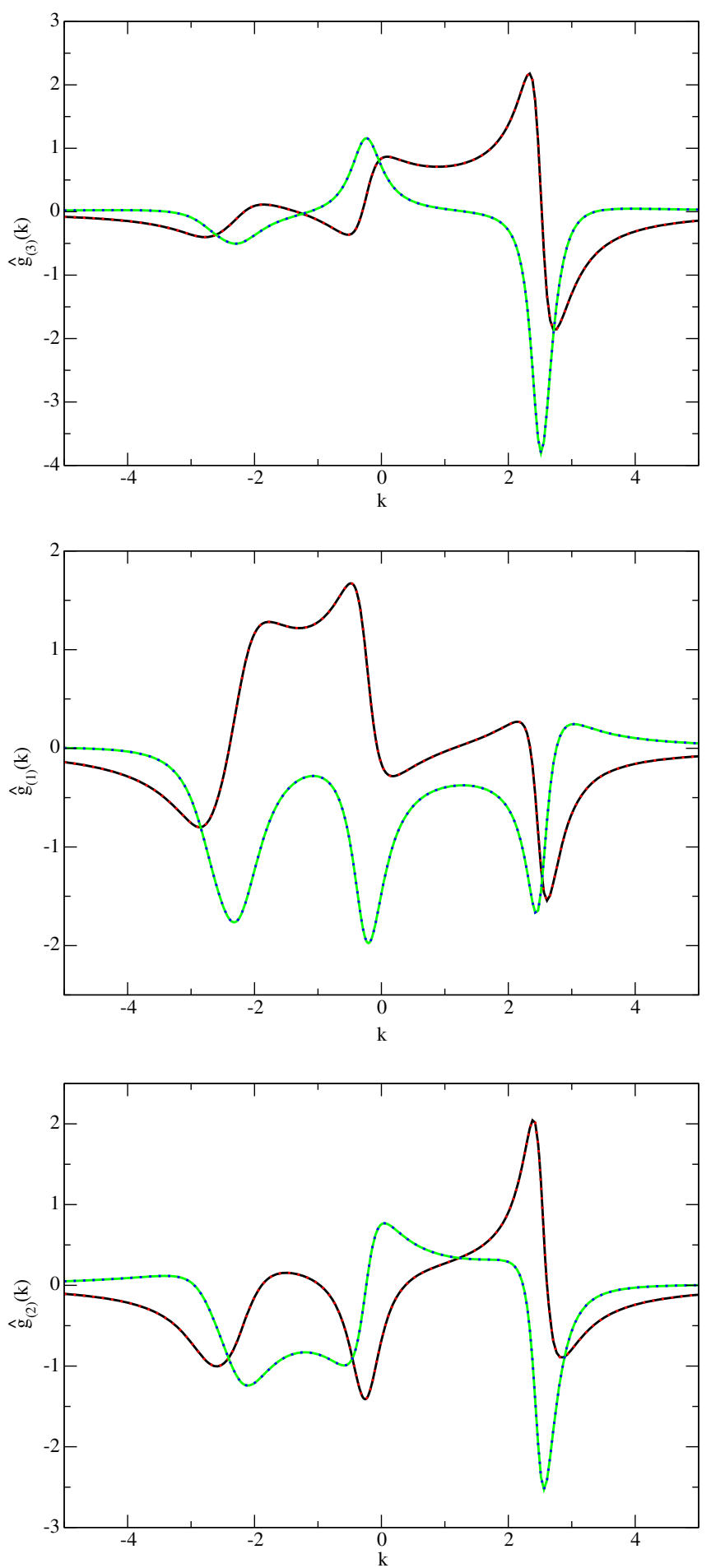

FIG. 5. The comparison of numerical solutions of integral Eq. (A23) with exact solutions given in Eqs. (A15)-(A17): the solutions of $\hat{g}_{(3,1,2)}$ are presented in upper, middle, and lower panels respectively. Solid black and solid green curves represent real and imaginary parts of numerical solutions, and dotted red and blue are real and imaginary parts of exact solutions respectively. The parameters of the toy model are chosen as $m V_{0}=2.0, q_{12}=$ $1.0+0.4 i$ and $q_{3}=2.5+0.2 i$, where an imaginary part is given to both $\left(q_{12}, q_{3}\right)$ to smooth out the curves near the pole position only for better visualization purposes.

$$
\begin{aligned}
\hat{g}_{(1)}^{(0)}\left(k ; q_{i j}, q_{k}\right)= & i \frac{2 q_{31} i t_{31}\left(-q_{31}\right)}{\left(k-q_{3}-i \epsilon\right)\left(k-q_{1}+i \epsilon\right)} \\
& -i \frac{2 q_{12} i t_{12}\left(q_{12}\right)}{\left(k-q_{2}-i \epsilon\right)\left(k-q_{1}+i \epsilon\right)}, \\
\hat{g}_{(2)}^{(0)}\left(k ; q_{i j}, q_{k}\right)= & -i \frac{2 q_{12} i t_{12}\left(q_{12}\right)\left[1+i t_{23}\left(-q_{23}\right)\right]}{\left(k-q_{2}-i \epsilon\right)\left(k-q_{1}+i \epsilon\right)} \\
& +i \frac{2 q_{23} i t_{23}\left(-q_{23}\right)}{\left(k-q_{3}-i \epsilon\right)\left(k-q_{2}-i \epsilon\right)} .
\end{aligned}
$$

Faddeev equations for $\hat{g}_{(\gamma)}$ 's, given by Eq. (A23), are solved numerically by the matrix inversion method, and the comparison of numerical solutions with exact solutions in Eqs. (A15)-(A17) is presented in Fig. 5.

\section{APPENDIX B: FADDEEV EQUATIONS INCLUDING THREE-BODY FORCE}

In previous sections, our discussion of three-body problem was restricted on the interactions of three particles with only pair-wise $\delta$-function potentials. In this section, we would like to extend our discussion of three-body interaction by including a spherical symmetric three-body force potential, $U(r)$, where $r=\sqrt{r_{\alpha \beta}^{2}+\frac{4}{3} r_{\gamma}^{2}}$, and give a brief presentation above how the three-body force may be handled in the Faddeev equations approach. By including a three-body force potential, $U(r)$, Schrödinger equation now has the form of

$$
\begin{aligned}
& {\left[-\frac{1}{2 m} \sum_{i=1}^{3} \frac{d^{2}}{d x_{i}^{2}}+\sum_{\gamma=1}^{3} V_{\alpha \beta} \delta\left(r_{\alpha \beta}\right)+U(r)-E\right]} \\
& \quad \times \Psi\left(x_{1}, x_{2}, x_{3} ; p_{1}, p_{2}, p_{3}\right)=0
\end{aligned}
$$

Let us consider the scattering of three-particle with an incoming wave of three free particles, $\Psi_{(0)}$. Three-body wave function may thus be expressed in the form of

$$
\Psi=\Psi_{(0)}+\sum_{\gamma=1}^{3} \Psi_{(\gamma)}+\Psi_{(U)},
$$

where $\Psi_{(\gamma)}$ satisfies equation,

$$
\begin{aligned}
& {\left[-\frac{1}{2 m} \sum_{i=1}^{3} \frac{d^{2}}{d x_{i}^{2}}+V_{\alpha \beta} \delta\left(r_{\alpha \beta}\right)-E\right] \Psi_{(\gamma)}} \\
& \quad=-V_{\alpha \beta} \delta\left(r_{\alpha \beta}\right)\left[\Psi_{(0)}+\Psi_{(\alpha)}+\Psi_{(\beta)}+\Psi_{(U)}\right], \\
& \gamma \neq \alpha \neq \beta,
\end{aligned}
$$

and similarly the equation for $\Psi_{(U)}$ is given by 


$$
\begin{aligned}
& {\left[-\frac{1}{2 m} \sum_{i=1}^{3} \frac{d^{2}}{d x_{i}^{2}}+U(r)-E\right] \Psi_{(U)}} \\
& =-U(r)\left[\Psi_{(0)}+\Psi_{(\alpha)}+\Psi_{(\beta)}+\Psi_{(\gamma)}\right]
\end{aligned}
$$

The Lippmann-Schwinger equation for relative wave function, $\psi_{(\gamma)}$ and $\psi_{(U)}$, can be obtained respectively as

$$
\begin{aligned}
\psi_{(\gamma)}\left(r_{\alpha \beta}, r_{\gamma} ; q_{i j}, q_{k}\right) & \\
= & \int_{-\infty}^{\infty} \frac{d k}{2 \pi} e^{i \sqrt{\sigma^{2}-\frac{3}{4} k^{2}} r_{\alpha \beta}} e^{i k r_{\gamma}} i t_{\alpha \beta}\left(\sqrt{\sigma^{2}-\frac{3}{4} k^{2}}\right) \\
& \times \int_{-\infty}^{\infty} d r_{\alpha \beta}^{\prime} d r_{\gamma}^{\prime} e^{-i k r_{\gamma}^{\prime}} \delta\left(r_{\alpha \beta}^{\prime}\right)\left[\psi_{(0)}\left(r_{\alpha \beta}^{\prime}, r_{\gamma}^{\prime} ; q_{i j}, q_{k}\right)\right. \\
& +\psi_{(\alpha)}\left(r_{\beta \gamma}^{\prime}, r_{\alpha}^{\prime} ; q_{i j}, q_{k}\right)+\psi_{(\beta)}\left(r_{\gamma \alpha}^{\prime}, r_{\beta}^{\prime} ; q_{i j}, q_{k}\right) \\
& \left.+\psi_{(U)}\left(r_{\alpha \beta}^{\prime}, r_{\gamma}^{\prime} ; q_{i j}, q_{k}\right)\right], \\
\alpha \neq & \neq \neq \neq \gamma,
\end{aligned}
$$

and

$$
\begin{aligned}
\psi_{(U)} & \left(r_{12}, r_{3} ; q_{i j}, q_{k}\right) \\
= & \int_{-\infty}^{\infty} d r_{12}^{\prime} d r_{3}^{\prime} G_{(U)}\left(r_{12}, r_{3} ; r_{12}^{\prime}, r_{3}^{\prime} ; \sigma\right) m U\left(r^{\prime}\right) \\
& \times\left[\psi_{(0)}\left(r_{12}^{\prime}, r_{3}^{\prime} ; q_{i j}, q_{k}\right)+\sum_{\gamma=1}^{3} \psi_{(\gamma)}\left(r_{\alpha \beta}^{\prime}, r_{\gamma}^{\prime} ; q_{i j}, q_{k}\right)\right],
\end{aligned}
$$$$
\alpha \neq \beta \neq \gamma \text {. }
$$

The Green's function, $G_{(U)}$, satisfies equation,

$$
\begin{aligned}
& {\left[\sigma^{2}+\frac{d^{2}}{d r_{12}^{2}}+\frac{3}{4} \frac{d^{2}}{d r_{3}^{2}}-m U(r)\right] G_{(U)}\left(r_{12}, r_{3} ; r_{12}^{\prime}, r_{3}^{\prime} ; \sigma\right)} \\
& \quad=\delta\left(r_{12}-r_{12}^{\prime}\right) \delta\left(r_{3}-r_{3}^{\prime}\right)
\end{aligned}
$$

Next, let us introduce the scattering amplitudes by

$$
\begin{aligned}
g_{(\gamma)}\left(k ; q_{i j}, q_{k}\right)= & \int_{-\infty}^{\infty} d r e^{-i k r}[\psi(\alpha) \\
& +\psi_{(\beta)}\left(r,-\frac{r}{2} ; q_{i j}, q_{k}\right) \\
& +\psi_{(U)}\left(0, r ; q_{i j}, q_{k}\right) \\
& \left.\left.=q_{k}\right)\right]
\end{aligned}
$$

$$
\begin{aligned}
T_{(\gamma)}\left(k ; q_{i j}, q_{k}\right)= & -\int_{-\infty}^{\infty} d r_{\alpha \beta} d r_{\gamma} e^{-i k r_{\gamma}} \\
& \times m V_{\alpha \beta} \delta\left(r_{\alpha \beta}\right) \psi\left(r_{\alpha \beta}, r_{\gamma} ; q_{i j}, q_{k}\right),
\end{aligned}
$$

$$
\begin{aligned}
T_{(U)}\left(k_{12}, k_{3} ; q_{i j}, q_{k}\right)= & -\int_{-\infty}^{\infty} d r_{12} d r_{3} e^{-i k_{12} r_{12}} e^{-i k_{3} r_{3}} \\
& \times m U(r) \psi\left(r_{12}, r_{3} ; q_{i j}, q_{k}\right) .
\end{aligned}
$$

The $T_{(\gamma)}$ - and $g_{(\gamma)}$-amplitudes are still related by Eq. (A8). The total three-body scattering amplitude with the presence of three-body force is thus given by

$$
\begin{aligned}
T & \left(k_{12}, k_{3} ; q_{i j}, q_{k}\right) \\
& =\sum_{\gamma=1}^{3} T_{(\gamma)}\left(k_{\gamma} ; q_{i j}, q_{k}\right)+T_{(U)}\left(k_{12}, k_{3} ; q_{i j}, q_{k}\right),
\end{aligned}
$$

where $k_{\alpha}=-k_{\alpha \beta}-\frac{k_{y}}{2}$ and $k_{\beta}=k_{\alpha \beta}-\frac{k_{\gamma}}{2}$.

The wave functions, $\psi_{(\gamma)}$ and $\psi_{(U)}$, are given in terms of $T$-amplitude by

$$
\begin{aligned}
& \psi_{(\gamma)}\left(r_{\alpha \beta}, r_{\gamma} ; q_{i j}, q_{k}\right) \\
& =i \int_{-\infty}^{\infty} \frac{d k}{2 \pi} \frac{e^{i \sqrt{\sigma^{2}-\frac{3}{4} k^{2}}\left|r_{\alpha \beta}\right|} e^{i k r_{\gamma}}}{2 \sqrt{\sigma^{2}-\frac{3}{4} k^{2}}} T_{(\gamma)}\left(k ; q_{i j}, q_{k}\right),
\end{aligned}
$$

$$
\begin{aligned}
& \psi_{(U)}\left(r_{12}, r_{3} ; q_{i j}, q_{k}\right) \\
& =-\int_{-\infty}^{\infty} \frac{d k_{12}}{2 \pi} \frac{d k_{3}}{2 \pi} \frac{e^{i k_{12} r_{12}} e^{i k_{3} r_{3}} T_{(U)}\left(k_{12}, k_{3} ; q_{i j}, q_{k}\right)}{\sigma^{2}-k_{12}^{2}-\frac{3}{4} k_{3}^{2}+i \epsilon} .
\end{aligned}
$$

Equations (B9)-(B13) yield a sets of coupled equations for $g_{(\gamma)}$ and $T_{(U)}$ amplitudes respectively,

$$
\begin{aligned}
g_{(\gamma)}\left(k ; q_{i j}, q_{k}\right)= & g_{(\gamma)}^{(0)}\left(k ; q_{i j}, q_{k}\right)-\int_{-\infty}^{\infty} \frac{d q}{2 \pi} \frac{T_{(U)}\left(q, k ; q_{i j}, q_{k}\right)}{\sigma^{2}-q^{2}-\frac{3}{4} k^{2}+i \epsilon} \\
& +i \int_{-\infty}^{\infty} \frac{d q}{2 \pi} \frac{2 \sqrt{\sigma^{2}-\frac{3}{4} q^{2}}}{\left(\sigma^{2}-\frac{3}{4} q^{2}\right)-\left(k+\frac{q}{2}\right)^{2}+i \epsilon} \\
& \times\left[i t_{\beta \gamma}\left(\sqrt{\sigma^{2}-\frac{3}{4} q^{2}}\right) g_{(\alpha)}\left(q ; q_{i j}, q_{k}\right)\right. \\
& \left.+i t_{\gamma \alpha}\left(\sqrt{\sigma^{2}-\frac{3}{4} q^{2}}\right) g_{(\beta)}\left(q ; q_{i j}, q_{k}\right)\right], \\
\alpha & \neq \beta \neq \gamma,
\end{aligned}
$$

where $g_{(\gamma)}^{(0)}$ is defined in Eq. (A7), and

$$
\begin{aligned}
& T_{(U)}\left(k_{12}, k_{3} ; q_{i j}, q_{k}\right) \\
& =v_{(U)}\left(k_{12}, k_{3} ; q_{i j}, q_{k}\right)+\sum_{\gamma=1}^{3} \int_{-\infty}^{\infty} \frac{d q}{2 \pi} \mathcal{F}_{(\alpha \beta)}\left(k_{12}, k_{3} ; q\right) \\
& \quad \times i t_{\alpha \beta}\left(\sqrt{\sigma^{2}-\frac{3}{4} q^{2}}\right) g_{(\gamma)}\left(q ; q_{i j}, q_{k}\right), \\
& \alpha \neq \beta \neq \gamma .
\end{aligned}
$$


The functions, $v_{(U)}$ and $\mathcal{F}_{(\alpha \beta)}$, are defined respectively by

$$
v_{(U)}\left(k_{12}, k_{3} ; q_{i j}, q_{k}\right)=-\int_{-\infty}^{\infty} d r_{12} d r_{3}\left[\psi_{(0)}\left(r_{12}, r_{3} ; q_{i j}, q_{k}\right)+\sum_{\gamma=1}^{3} \psi_{(\gamma)}^{(i n)}\left(r_{\alpha \beta}, r_{\gamma} ; q_{i j}, q_{k}\right)\right] m U(r) \phi_{(U)}^{*}\left(r_{12}, r_{3} ; k_{12}, k_{3}\right) \text {, }
$$

and

$$
\mathcal{F}_{(\alpha \beta)}\left(k_{12}, k_{3} ; q\right)=-\int_{-\infty}^{\infty} d r_{12} d r_{3} e^{i \sqrt{\sigma^{2}-\frac{3}{4} q^{2}}\left|r_{\alpha \beta}\right|} e^{i q r_{\gamma}} m U(r) \phi_{(U)}^{*}\left(r_{12}, r_{3} ; k_{12}, k_{3}\right),
$$

where

$$
\psi_{(\gamma)}^{(i n)}\left(r_{\alpha \beta}, r_{\gamma} ; q_{i j}, q_{k}\right)=\int_{-\infty}^{\infty} \frac{d k}{2 \pi} e^{i \sqrt{\sigma^{2}-\frac{3}{4} k^{2}}\left|r_{\alpha \beta}\right|} e^{i k r_{\gamma}} i t_{\alpha \beta}\left(\sqrt{\sigma^{2}-\frac{3}{4} k^{2}}\right) \int_{-\infty}^{\infty} d r_{\gamma}^{\prime} e^{-i k r_{\gamma}^{\prime}} \psi(0)\left(0, r_{\gamma}^{\prime} ; q_{i j}, q_{k}\right)
$$

and

$$
\phi_{(U)}^{*}\left(r_{12}, r_{3} ; k_{12}, k_{3}\right)=e^{-i k_{12} r_{12}} e^{-i k_{3} r_{3}}+\int_{-\infty}^{\infty} d r_{12}^{\prime} d r_{3}^{\prime} e^{-i k_{12} r_{12}^{\prime}} e^{-i k_{3} r_{3}^{\prime}} m U\left(r^{\prime}\right) G_{(U)}\left(r_{12}^{\prime}, r_{3}^{\prime} ; r_{12}, r_{3} ; \sigma\right)
$$

The wave function $\phi_{(U)}$ satisfies Schrödinger equation with the presence of three-body forces potential alone,

$$
\left[\sigma^{2}+\frac{d^{2}}{d r_{12}^{2}}+\frac{3}{4} \frac{d^{2}}{d r_{3}^{2}}-m U(r)\right] \phi_{(U)}\left(r_{12}, r_{3} ; q_{i j}, q_{k}\right)=0
$$

The finite volume three-body wave function again can be constructed from three-body free space wave function, see Eq. (9), therefore, when three-body force is considered, we obtain the finite volume three-body wave function,

$$
\psi^{(L)}\left(r_{12}, r_{3} ; q_{i j}, q_{k}\right)=\sum_{\gamma=1}^{3} \psi_{(\gamma)}^{(L)}\left(r_{\alpha \beta}, r_{\gamma} ; q_{i j}, q_{k}\right)-\frac{1}{L^{2}} \sum_{\left(n_{12}, n_{3}\right) \in \mathbb{Z}}^{\substack { k_{12}=-\frac{P}{3}-\frac{k_{3}}{2}+\frac{2 \pi}{L} n_{12} \\
\begin{subarray}{c}{k_{3}=-\frac{2 P}{2}+\frac{2 \pi}{n_{3}} \\
i k_{12} r_{12}{ k _ { 1 2 } = - \frac { P } { 3 } - \frac { k _ { 3 } } { 2 } + \frac { 2 \pi } { L } n _ { 1 2 } \\
\begin{subarray} { c } { k _ { 3 } = - \frac { 2 P } { 2 } + \frac { 2 \pi } { n _ { 3 } } \\
i k _ { 1 2 } r _ { 1 2 } } }\end{subarray}} e^{i k_{3} r_{3}} \frac{T_{(U)}\left(k_{12}, k_{3} ; q_{i j}, q_{k}\right)}{\sigma^{2}-k_{12}^{2}-\frac{3}{4} k_{3}^{2}+i \epsilon}
$$

where $\psi_{(\gamma)}^{(L)}$ is given by Eq. (11).

[1] J. Kambor, C. Wiesendanger, and D. Wyler, Nucl. Phys. B465, 215 (1996).

[2] A. V. Anisovich and H. Leutwyler, Phys. Lett. B 375, 335 (1996).

[3] G. Colangelo, S. Lanz, and E. Passemar, Proc. Sci., CD09 (2009) 047.

[4] S. Lanz, Proc. Sci., CD12 (2013) 007.

[5] S. P. Schneider, B. Kubis, and C. Ditsche, J. High Energy Phys. 02 (2011) 028.

[6] K. Kampf, M. Knecht, J. Novotny, and M. Zdrahal, Phys. Rev. D 84, 114015 (2011).

[7] P. Guo, I. V. Danilkin, D. Schott, C. Fernández-Ramírez, V. Mathieu, and A. P. Szczepaniak, Phys. Rev. D 92, 054016 (2015).
[8] P. Guo, I. V. Danilkin, C. Fernández-Ramírez, V. Mathieu, and A. P. Szczepaniak, Phys. Lett. B 771, 497 (2017).

[9] J. G. Taylor, Phys. Rev. 150, 1321 (1966).

[10] J.-L. Basdevant and R. E. Kreps, Phys. Rev. 141, 1398 (1966).

[11] F. Gross, Phys. Rev. C 26, 2226 (1982).

[12] L. D. Faddeev, Zh. Eksp. Teor. Fiz. 39, 1459 (1960) [Sov. Phys.-JETP 12, 1014 (1961)].

[13] L. D. Faddeev, Mathematical Aspects of the Three-Body Problem in the Quantum Scattering Theory (Israel Program for Scientific Translation, Jerusalem, Israel, 1965).

[14] W. Glöckle, The Quantum Mechanical Few-Body Problem (Springer, Berlin, 1983).

[15] A. C. Phillips, Phys. Rev. 142, 984 (1966). 
[16] D. V. Fedorov and A S. Jensen, Phys. Rev. Lett. 71, 4103 (1993).

[17] W. Glöckle, H. Witala, D. Hüber, H. Kamada, and J. Golak, Phys. Rep. 274, 107 (1996).

[18] N. N. Khuri and S. B. Treiman, Phys. Rev. 119, 1115 (1960).

[19] J. B. Bronzan and C. Kacser, Phys. Rev. 132, 2703 (1963).

[20] I. J. R. Aitchison, II Nuovo Cimento 35, 434 (1965).

[21] I. J. R. Aitchison, Phys. Rev. 137, B1070 (1965); 154, 1622 (1967).

[22] I. J. R. Aitchison and R. Pasquier, Phys. Rev. 152, 1274 (1966).

[23] R. Pasquier and J. Y. Pasquier, Phys. Rev. 170, 1294 (1968).

[24] R. Pasquier and J. Y. Pasquier, Phys. Rev. 177, 2482 (1969).

[25] P. Guo, I. V. Danilkin, and A. P. Szczepaniak, Eur. Phys. J. A 51, 135 (2015).

[26] P. Guo, Phys. Rev. D 91, 076012 (2015).

[27] I. V. Danilkin, C. Fernández-Ramírez, P. Guo, V. Mathieu, D. Schott, and A. P. Szczepaniak, Phys. Rev. D 91, 094029 (2015).

[28] P. Guo, Mod. Phys. Lett. A 31, 1650058 (2016).

[29] S. Aoki et al. (CP-PACS Collaboration), Phys. Rev. D 76, 094506 (2007).

[30] K. Sasaki and N. Ishizuka, Phys. Rev. D 78, 014511 (2008).

[31] X. Feng, K. Jansen, and D. B. Renner, Phys. Rev. D 83, 094505 (2011).

[32] J. J. Dudek, R. G. Edwards, M. J. Peardon, D. G. Richards, and C. E. Thomas (Hadron Spectrum Collaboration), Phys. Rev. D 83, 071504 (2011).

[33] S. R. Beane, E. Chang, W. Detmold, H. W. Lin, T. C. Luu, K. Orginos, A. Parreño, M. J. Savage, A. Torok, and A. Walker-Loud (NPLQCD Collaboration), Phys. Rev. D 85, 034505 (2012).

[34] C. B. Lang, D. Mohler, S. Prelovsek, and M. Vidmar, Phys. Rev. D 84, 054503 (2011).

[35] S. Aoki et al. (CS Collaboration), Phys. Rev. D 84, 094505 (2011).

[36] J. J. Dudek, R. G. Edwards, and C. E. Thomas (Hadron Spectrum Collaboration), Phys. Rev. D 86, 034031 (2012).

[37] J. J. Dudek, R. G. Edwards, and C. E. Thomas, Phys. Rev. D 87, 034505 (2013).

[38] D. J. Wilson, J. J. Dudek, R. G. Edwards, and C. E. Thomas, Phys. Rev. D 91, 054008 (2015).

[39] D. J. Wilson, R. A. Briceno, J. J. Dudek, R. G. Edwards, and C. E. Thomas, Phys. Rev. D 92, 094502 (2015).

[40] J. J. Dudek, R. G. Edwards, and D. J. Wilson (Hadron Spectrum Collaboration), Phys. Rev. D 93, 094506 (2016).

[41] M. Lüscher, Nucl. Phys. B354, 531 (1991).

[42] K. Rummukainen and S. Gottlieb, Nucl. Phys. B450, 397 (1995).

[43] C.-J. D. Lin, G. Martinelli, C. T. Sachrajda, and M. Testa, Nucl. Phys. B619, 467 (2001).

[44] N. H. Christ, C. Kim, and T. Yamazaki, Phys. Rev. D 72, 114506 (2005).

[45] V. Bernard, U.-G. Meißner, and A. Rusetsky, Nucl. Phys. B788, 1 (2008).

[46] V. Bernard, M. Lage, U.-G. Meißner, and A. Rusetsky, J. High Energy Phys. 08 (2008) 024.

[47] S. He, X. Feng, and C. Liu, J. High Energy Phys. 07 (2005) 011.
[48] M. Lage, U.-G. Meißner, and A. Rusetsky, Phys. Lett. B 681, 439 (2009).

[49] M. Döring, U.-G. Meißner, E. Oset, and A. Rusetsky, Eur. Phys. J. A 47, 139 (2011).

[50] S. Aoki, N. Ishii, T. Doi, T. Hatsuda, Y. Ikeda, T. Inoue, K. Murano, H. Nemura, and K. Sasaki (HAL QCD Collaboration), Proc. Jpn. Acad. Ser. B 87, 509 (2011).

[51] R. A. Briceno and Z. Davoudi, Phys. Rev. D 88, 094507 (2013).

[52] M. T. Hansen and S. R. Sharpe, Phys. Rev. D 86, 016007 (2012).

[53] P. Guo, J. Dudek, R. Edwards, and A. P. Szczepaniak, Phys. Rev. D 88, 014501 (2013).

[54] P. Guo, Phys. Rev. D 88, 014507 (2013).

[55] S. M. Reimann and M. Manninen, Rev. Mod. Phys. 74, 1283 (2002).

[56] J. H. Jefferson, M. Fearn, D. L. J. Tipton, and T. P. Spiller, Phys. Rev. A 66, 042328 (2002).

[57] V. N. Golovach, A. Khaetskii, and D. Loss, Phys. Rev. B 77, 045328 (2008)

[58] S. Schröter, P.-A. Hervieux, G. Manfred, J. Eiglsperger, and J. Madroñero, Phys. Rev. B 87, 155413 (2013).

[59] T. Frostad, J. P. Hansen, C. J. Wesslën, E. Lindroth, and E. Rasanen, Eur. Phys. J. B 86, 430 (2013).

[60] J. Sarkka and A. Harju, Physica E (Amsterdam) 42, 844 (2010).

[61] Z.-W. Wang and S.-S. Li, Solid State Commun. 151, 1667 (2011).

[62] J. S. Ahn, Superlattices Microstruct. 65, 113 (2014).

[63] M. Mengesha and V. N. Mal'nev, Ukr. J. Phys. 56, 1228 (2011).

[64] R. Khordad, Superlattices Microstruct. 62, 166 (2013).

[65] M. R. Fulla, F. Rodriguez-Prada, and J. H. Marn Cadavid, Superlattices Microstruct. 49, 252 (2011).

[66] L. F. Garcia, W. Gutirrez, and I. D. Mikhailov, Physica B (Amsterdam) 455, 14 (2014).

[67] Y. Yakar, B. akir, and A. Özmen, Comput. Phys. Commun. 188, 88 (2015).

[68] B. Gakir, Y. Yakar, and A. Özmen, Physica B Condens. Matter 458, 138 (2015).

[69] A. Aharony, O. Entin-Wohlman, Y. Levinson, and Y. Imry, Ann. Phys. (Berlin) 8, 685 (1999).

[70] B. L. Altshuler and A. G. Aronov, Modern Condens. Matter Sci. 10, 1 (1985).

[71] D. A. Huse, R. Nandkishore, and V. Oganesyan, Phys. Rev. B 90, 174202 (2014).

[72] A. M. Somoza, M. Ortuño, V. Gasparian, and M. Piño, J. Phys. Condens. Matter 27, 335503 (2015).

[73] S. Kreuzer and H.-W. Hammer, Phys. Lett. B 673, 260 (2009).

[74] S. Kreuzer and H.-W. Hammer, Eur. Phys. J. A 43, 229 (2010).

[75] S. Kreuzer and H.-W. Hammer, Eur. Phys. J. A 48, 93 (2012).

[76] K. Polejaeva and A. Rusetsky, Eur. Phys. J. A 48, 67 (2012).

[77] R. A. Briceno and Z. Davoudi, Phys. Rev. D 87, 094507 (2013).

[78] M. T. Hansen and S. R. Sharpe, Phys. Rev. D 90, 116003 (2014).

[79] M. T. Hansen and S. R. Sharpe, Phys. Rev. D 92, 114509 (2015). 
[80] M. T. Hansen and S. R. Sharpe, Phys. Rev. D 93, 096006 (2016).

[81] H.-W. Hammer, J.-Y. Pang, and A. Rusetsky, J. High Energy Phys. 09 (2017) 109.

[82] H.-W. Hammer, J.-Y. Pang, and A. Rusetsky, J. High Energy Phys. 10 (2017) 115.

[83] P. Guo, Phys. Rev. D 95, 054508 (2017).

[84] P. Guo and V. Gasparian, Phys. Lett. B 774, 441 (2017).

[85] U.-G. Meißner, G. Rios, and A. Rusetsky, Phys. Rev. Lett. 114, 091602 (2015); 117, 069902(E) (2016).
[86] R. A. Briceno, M. T. Hansen, and S. R. Sharpe, Phys. Rev. D 95, 074510 (2017).

[87] S. R. Sharpe, Phys. Rev. D 96, 054515 (2017).

[88] M. Mai and M. Döring, arXiv:1709.08222.

[89] J. B. McGuire, J. Math. Phys. (N.Y.) 5, 622 (1964).

[90] J. B. McGuire and C. A. Hurst, J. Math. Phys. (N.Y.) 29, 155 (1988).

[91] L. R. Dodd, J. Math. Phys. (N.Y.) 11, 207 (1970).

[92] C. K. Majumdar, J. Math. Phys. (N.Y.) 13, 705 (1972). 